University of Nebraska - Lincoln

DigitalCommons@University of Nebraska - Lincoln

4-2011

\title{
Self-Assembled Nanometer-Scale Magnetic Networks on Surfaces: Fundamental Interactions and Functional Properties
}

\author{
Carlo Carbone \\ Consiglio Nazionale delle Ricerche, Trieste, Italy, carlo.carbone@trieste.ism.cnr.it \\ Sandra Gardonio \\ Consiglio Nazionale delle Ricerche, Trieste, Italy \\ Paolo Moras \\ Consiglio Nazionale delle Ricerche, Trieste, Italy, paolo.moras@ism.cnr.it \\ Samir Lounis \\ Institut für Festkörperforschung, Germany, s.lounis@fz-juelich.de \\ Marcus Heide \\ Max-Planck-Institut für Festkörperforschung \\ See next page for additional authors \\ Follow this and additional works at: https://digitalcommons.unl.edu/physicsenders \\ Part of the Physics Commons
}

Carbone, Carlo; Gardonio, Sandra; Moras, Paolo; Lounis, Samir; Heide, Marcus; Bihlmayer, Gustav; Atodiresei, Nicolae; Dederichs, Peter Heinz; Blügel, Stefan; Vlaic, Sergio; Lehnert, Anne; Ouazi, Safia; Rusponi, Stefano; Brune, Harald; Honolka, Jan; Enders, Axel; Kern, Klaus; Stepanow, Sebastian; Krull, Cornelius; Balashov, Timofey; Mugarza, Aitor; and Gambardella, Pietro, "Self-Assembled Nanometer-Scale Magnetic Networks on Surfaces: Fundamental Interactions and Functional Properties" (2011). Axel Enders Publications. 32.

https://digitalcommons.unl.edu/physicsenders/32

This Article is brought to you for free and open access by the Research Papers in Physics and Astronomy at DigitalCommons@University of Nebraska - Lincoln. It has been accepted for inclusion in Axel Enders Publications by an authorized administrator of DigitalCommons@University of Nebraska - Lincoln. 


\section{Authors}

Carlo Carbone, Sandra Gardonio, Paolo Moras, Samir Lounis, Marcus Heide, Gustav Bihlmayer, Nicolae Atodiresei, Peter Heinz Dederichs, Stefan Blügel, Sergio Vlaic, Anne Lehnert, Safia Ouazi, Stefano Rusponi, Harald Brune, Jan Honolka, Axel Enders, Klaus Kern, Sebastian Stepanow, Cornelius Krull, Timofey

Balashov, Aitor Mugarza, and Pietro Gambardella 


\title{
Self-Assembled Nanometer-Scale Magnetic Networks on Surfaces: Fundamental Interactions and Functional Properties
}

\author{
Carlo Carbone, Sandra Gardonio, Paolo Moras, Samir Lounis, Marcus Heide, Gustav Bihlmayer, \\ Nicolae Atodiresei, Peter Heinz Dederichs, Stefan Blügel, Sergio Vlaic, Anne Lehnert, Safia Ouazi, \\ Stefano Rusponi, Harald Brune, Jan Honolka, Axel Enders, Klaus Kern, Sebastian Stepanow, \\ Cornelius Krull, Timofey Balashov, Aitor Mugarza, and Pietro Gambardella
}

\begin{abstract}
Nanomagnets of controlled size, organized into regular patterns open new perspectives in the fields of nanoelectronics, spintronics, and quantum computation. Self-assembling processes on various types of substrates allow designing fine-structured architectures and tuning of their magnetic properties. Here, starting from a description of fundamental magnetic interactions at the nanoscale, we review recent experimental approaches to fabricate zero-, one-, and two-dimensional magnetic particle arrays with dimensions reduced to the atomic limit and unprecedented areal density. We describe systems composed of individual magnetic atoms, metal-organic networks, metal wires, and bimetallic particles, as well as strategies to control their magnetic moment, anisotropy, and temperature-dependent magnetic behavior. The investigation of self-assembled subnanometer magnetic particles leads to significant progress in the design of fundamental and functional aspects, mutual interactions among the magnetic units, and their coupling with the environment.
\end{abstract}

Keywords: nanomaterials, magnetism, magnetic materials, self-assembly, networks

\section{Introduction}

Nanomagnets made of a few atoms and organized into regular patterns offer the possibility to extrapolate established principles of magnetic storage to novel materials for magnetoelectronics and quantum computation. ${ }^{1,2}$ Self-assembled magnetic arrays made of extremely small, almost identical units make it possible to attain larger areal densities compared to top-down fabrication methods by means of low-cost parallel deposition processes. In the nanometersize range, the susceptibility, saturation magnetization, and magnetic anisotropy can be tuned by tiny changes of the sys- tem dimensions and coupling to the environment. Quantum magnetic systems that interact weakly with the supporting substrate may inspire novel strategies in the fabrication of nanometer-scale, solid-state qubit ensembles. Here, we focus on developing new self-assembly strategies to build magnetic networks, aiming at fabricating atomic and subnanometric scale arrays of magnetic particles and molecules with designed functional properties. The miniaturization of finely structured magnetic elements requires a particularly demanding control of self-assembly processes as well as a detailed knowledge about the fundamental physical properties of the single particles. ${ }^{3}$ Progress in this field necessarily re-
Dr. C. Carbone, Dr. S. Gardonio, [+] Dr. P. Moras

Istituto di Struttura della Materia

Consiglio Nazionale delle Ricerche

Trieste, 34149, Italy

E-mail: carlo.carbone@trieste.ism.cnr.it

Dr. S. Lounis, Dr. M. Heide, Dr. G. Bihlmayer, Dr. N. Atodiresei, Prof. P. H. Dederichs, Prof. S. Blügel

Institut für Festkörperforschung \& Institute for Advanced Simulation

Forschungszentrum Jülich \& JARA

Jülich, 52425, Germany

S. Vlaic, Dr. A. Lehnert, Dr. S. Ouazi, Dr. S. Rusponi, Prof. H. Brune Institute of Condensed Matter Physics

Ecole Polytechnique Fédérale de Lausanne

Lausanne, 1015, Switzerland

Dr. J. Honolka, Prof. A. Enders, Prof. K. Kern

Max-Planck-Institute for Solid State Research

Stuttgart, 70569, Germany

\author{
Dr. S. Stepanow, C. Krull, Dr. T. Balashov, Dr. A. Mugarza \\ Catalan Institute of Nanotechnology (ICN-CIN2) \\ Barcelona, 08193, Spain \\ Prof. P. Gambardella \\ Catalan Institute of Nanotechnology (ICN-CIN2) \\ Barcelona, 08193, Spain \\ Prof. P. Gambardella \\ Institució Catalana de Recerca i Estudis Avançats (ICREA), \& Departa- \\ ment de Física \\ Universitat Autonoma de Barcelona \\ Barcelona, 08193, Spain
}

[ + ] Present address of Paolo Moras: University of Nova Gorica, Campus Ajdovscina

Vipavska Ajdovscina, 5270, Slovenia 
lies on both theoretical and experimental methods in order to understand how relevant magnetic properties are modified by varying the size, shape, and chemical composition of the individual particles. Moreover, the mutual interactions within the nanoelement network and with the supporting substrate constitute another important aspect that needs to be considered, as it contributes to define the overall magnetic behavior of self-assembled superstructures.

Magnetic interactions in self-assembled nanostructures can be very different from what is known from bulk magnets: large spatial separations of magnetic entities lead to weak, Ruderman-Kittel-Kasuya-Yosida (RKKY)-type interactions that are determined by the (nonmagnetic) substrate, weak spin-orbit coupling (SOC) effects can compete with exchange interactions, and the magnetic anisotropy energy becomes a main factor determining the stability of the magnetic structure (see Figure 1). One of the main challenges in engineering functional magnetic networks consists in obtaining particles sufficiently stable with respect to quantum or thermally activated magnetic fluctuations. ${ }^{4}$ This is a key issue as it directly reflects itself on the ultimate density limit with which one may store magnetic information at room temperature. The fundamental mechanisms defining the orientation and the stability of the magnetization in nanomagnets are extremely sensitive to the reduced coordination and low symmetry on the atomic scale. The stability of the magnetization is largely determined by the magneto-crystalline anisotropy, which depends on the symmetry of the atomic structure and on spin-orbit effects in the electronic bands, rather than by the dipolar interactions as in macroscopic and in micromagnetic systems. It was recently found that the magneto-crystalline anisotropy energy (MAE) of atomicscale systems is $10^{2}$ to $10^{3}$ times larger than the corresponding bulk values. ${ }^{5,6}$ Detailed control on self-assembly mechanisms allows to explore how the MAE and other magnetic properties such as the exchange-coupling strength, magnetic ordering, spin and orbital moments can be modified by varying the materials and the local atomic arrangement.

We show in this paper that by optimizing the strategy of fabrication methods it is possible to build model magnetic networks with unprecedented high areal or linear density constituted by molecular complexes, atomic chains, and small clusters with typical size and relative separation in the nanometer range. Our approach relies on self-organization processes following metal and molecular-beam deposition on

SELF-ASSEMBLED 2D, 1D, OD STRUCTURES

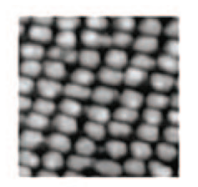

$5 \mathrm{~nm}$

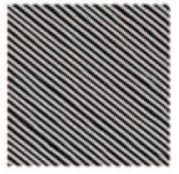

$2 \mathrm{~nm}$

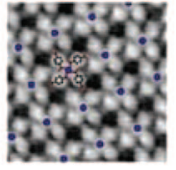

$1 \mathrm{~nm}$

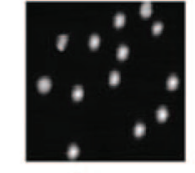

$0.1 \mathrm{~nm}$ -e'correlation exchange

MAGNETIC INTERACTIONS

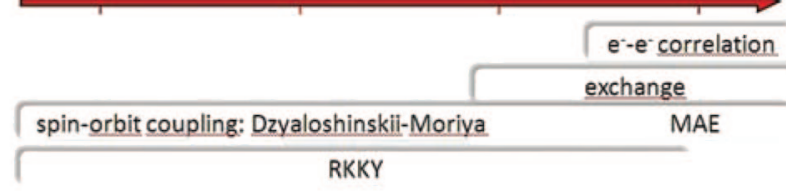

Figure 1. Schematics of magnetic structures that can be fabricated by self-assembly at or below the nanometer scale. The numbers in red indicate the average dimensions of the periodic unit cell. The range of relevant magnetic interactions is also shown.
Carlo Carbone leads the Trieste Section of the Istituto di Struttura della Materia of the Consiglio Nazionale delle Ricerche. He is an expert in the field of magnetism and electronic properties of low-dimensional structures and nanomaterials. His activity is largely based on experimental studies with advanced synchrotron techniques.

nanopatterned templates, exploiting the site-selective nucleation on substrates with periodic arrays of steps, dislocations, or molecules in order to obtain regular assemes of defined geometry and size with areal density up to 24 Tera units $i^{-2}$. We emphasize here the critical dependence of the magnetic behavior on the atomic coordination in different environments and geometries as well as methods to control the former. Ab initio electronic structure calare employed to highlight fundamental magnetic Experimental investigations rely on complementary tech that give access to both the structure and electronic croscopy (STM), photoemission spectroscopy, X-ray magnetic circular dichroism (XMCD), the and magneto-optic Kerr effect (MOKE). This paper is organized as follows: Section 2 outlines some of the basic interactions in low-dimensional structures and describes how they act to define particular magnetic structures and anisotropy properties of nanosystems. Specifically, the role of the RKKY and Dzyaoshinskii-Moriya interactions (DMI) in surface structures is in Sections 2.1 and 2.2 using a first-principles approach. Section 2.3 deals with the possibility of controlling the magnetic anisotropy of simple metal-organic molecules by means of charging, i.e., through oxidation/reduction rections. The interaction of magnetic atoms with nonmagnetic substrates is treated in Section 3 from an experimental point of view, focusing on the competition between electron correlation and hybridization effects that ultimately determine whether a system will be magnetic. The survival of sizable local magnetic moments is a prerequisite to obtain planar arrays of magnetic nanostructures by self-assembly supported on nonmagnetic metals. Sections 4-6 are dedicated to proper self-assembly processes: the zero-dimensional (0D) case of Fe atoms embedded into a square planar supramolecular network is presented in Section 4, where it is shown that the electronic structure and magnetic properties of the Fe adatoms can be steered through lateral and apical ligand coordination, progressively reducing hybridization with the metallic substrate and leading 
to a spin reorientation transition. Section 5 treats the case of 1D metal chains self-assembled on vicinal surface templates, discussing the emergence of complex magnetic configuration that result from the interplay of atomic coordination effects, $3 d-5 d$ alloying, and DMI. Finally, in Section 6 we show that 2D superlattices of magnetic islands with unprecedented areal density and narrow size distribution can be fabricated on natural patterns formed by the intersection of surface dislocation lines with closely spaced parallel steps. The magnetic nanoislands are monodomain and present a size (number of atoms) to blocking temperature ratio smaller than 5, which is $30 \%$ lower than previously achieved in similar structures. Strategies to enhance the structural and magnetic quality of such arrays are also discussed.

\section{Magnetic Interactions in Low Dimensions: Fermi Surface and Spin-Orbit Coupling Effects}

The miniaturization of magnetic structures brings new challenges for the stability of both the intrinsic and collective magnetic properties with respect to external perturbations, e.g., caused by temperature or external magnetic fields. The fact that it is possible to stabilize (at least locally) magnetic order in small structures can only be explained by changes of the material properties when the dimensionality of the system is reduced. Magnetic interactions in nanostructures can be considerably enhanced compared to macroscopic samples and, even more importantly in some cases, interactions forbidden by symmetry in extended 3D systems can suddenly become relevant. In this chapter, we show how fundamental magnetic interactions (RKKY, SOC, and orbital mediated exchange) in supported nanostructures can be assessed with the help of calculations based on density functional theory (DFT), providing guidelines for the engineering of novel magnetic materials at or below the nanoscale.

\subsection{RKKY Interactions Among Magnetic Atoms at the In- terface with a Nonmagnetic Metal}

Scalar interactions between magnetic moments or spins in a magnetic structure, i.e., the coupling of spins $\boldsymbol{S}_{i}$ and $\mathbf{S}_{j}$ by a term $J_{i j}\left(\mathbf{S}_{i} \cdot \mathbf{S}_{j}\right)$, can be mediated by long-ranged RKKY interactions ${ }^{7}$ via a metallic, nonmagnetic host material or substrate. For the spins in a self-assembled structure of magnetic particles, this indirect exchange interaction is essential for the magnetic order. These scalar interactions are rotational invariant in real space, i.e., without further magnetic couplings the orientation of the magnetic moments is independent of the spatial orientation of the crystal lattice. Already in the 1960s, Roth and co-workers ${ }^{8}$ tackled the task of describing RKKY interactions mediated by (anisotropic) Fermi surfaces. This theory forms the basis of our understanding on the oscillating periods of the interlayer magnetic exchange coupling ${ }^{9-11}$ for arbitrary Fermi surfaces. Using firstprinciples calculations, we demonstrate that, due to Fermi surfaces characteristics, bulk electrons scatter at impurities buried below a surface and can get strongly focused along anisotropic confined beams. ${ }^{12}$ Thus, the shape of the Fermi surface of a substrate can have far-reaching consequences, e.g., it can lead to a strong directional dependence of the indirect exchange interaction of adatoms on surfaces. ${ }^{13,14}$

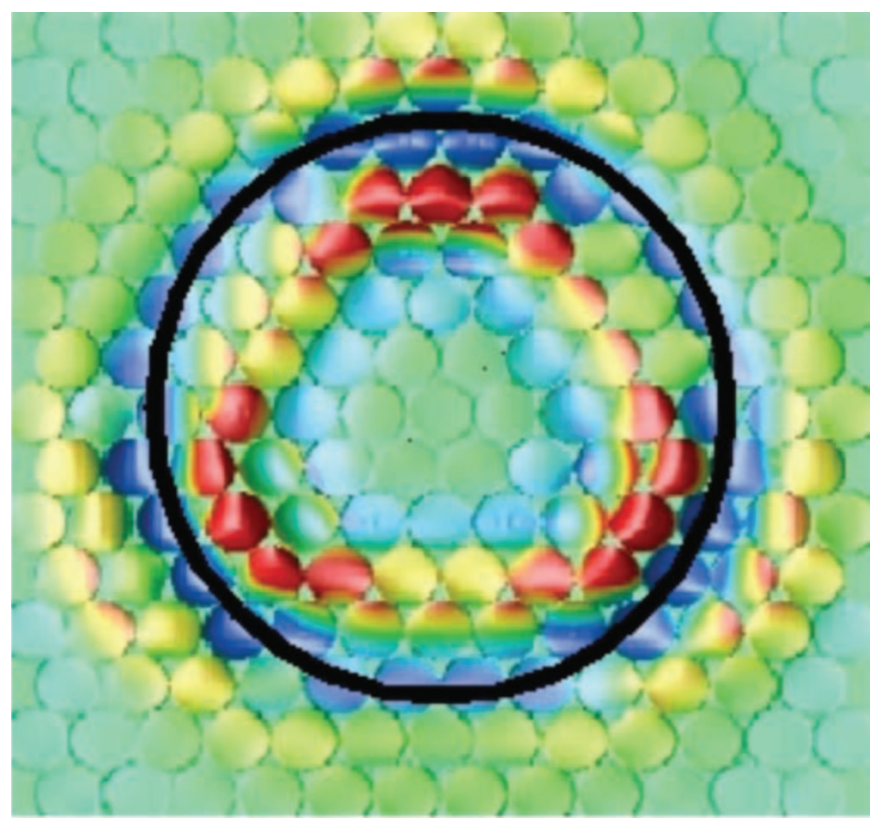

Figure 2. Impurity induced charge density at $E_{\mathrm{F}}$ after removing the ideal surface background charge (which includes the surface state) at a height of $\sim 6.1 \AA$ above the $\mathrm{Cu}(111)$ surface with an impurity sitting in the sixth layer below the surface. The triangular shape of the charge variation is in total accordance with the experiment of Weismann et al. ${ }^{12}$ and is explained by the flat areas of the Fermi surface of bulk $\mathrm{Cu}$. In black is the theoretical circle having a diameter calculated as $2 Z$ $\tan (\beta / 2)$ where $Z$ is the depth of the impurity and $\beta$ is the angle between focusing beams as deduced from the Fermi surface. The results fit well with the charge variation in the vacuum above an impurity sitting at $\sim 12.15 \AA$ below the surface. Adapted with permission. ${ }^{12}$ Copyright (C) 2009 American Association for the Advancement of Science.

Recently, we have shown by ab initio calculation combined with STM observations ${ }^{12}$ that anisotropic oscillations can be observed on top of $\mathrm{Cu}(111)$ or $\mathrm{Cu}(001)$ surfaces due to the presence of buried Co impurities. These anisotropic ripples show that the usual isotropic free-electron model is not valid in such real situations. We demonstrated that these intriguing features are nothing else than a visualization in real space of parts of the bulk copper Fermi surface that are relatively flat. This type of simulation requires a real-space investigation that was performed with the full potential Korringa-Kohn-Rostoker Green function method. ${ }^{15}$ Since we are interested in comparison to STM images, we use the Tersoff-Hamann model ${ }^{16}$ stating that scanning tunneling spectra can be related to the local density of states (LDOS) in a certain energy interval in the vacuum.

Figure 2 shows an example of the results of our simulations: the case of a Co impurity sitting at the $6^{\text {th }}$ layer below the $\mathrm{Cu}(111)$ surface $(\sim 12.15 \AA$ A below the surface). The charge induced in the vacuum has been computed up to an area of $30 \times 30 \AA^{2}$ above the impurity. One notices the triangular shape of the induced charge with high values at the corners of the triangle. In addition, a one and a half period ripple can be observed to oscillate from the red positive value to the blue negative values and finally to the almost zero green values.

The same period was also noticed in the STM experiment. ${ }^{12}$ To understand such a phenomenon, we start by giv- 
ing the form of the induced variation of the LDOS in the vacuum at position 0 by some buried Co impurity sitting at a position defined by $\mathbf{R}$ :

$$
\begin{aligned}
& \lim _{x \rightarrow 2} \Delta \operatorname{LDOS}_{\text {vacuum }}(0, \varepsilon) \\
& \quad=-1 / \pi \mathfrak{F} \iint \mathrm{d} \mathbf{r d} \mathbf{r}^{\prime}\left(G_{0}(0, \mathbf{r}, \varepsilon) t\left(\mathbf{r}, \mathbf{r}^{\prime} ; \varepsilon\right) G_{0}\left(\mathbf{r}^{\prime}, 0 ; \varepsilon\right)\right.
\end{aligned}
$$

where the Green function, $G_{0}$, is obtained from the KKR method, $t$ is the $t$-matrix corresponding to the potential change $V_{\text {new }}-V_{\text {host }}$ due to the impurity, and the radial integrations are performed within the impurity atomic cell. At very large distances $R$ between the impurity and the vacuum site one can apply the stationary phase approximation and end up with a result similar to that of the well-know theory of interlayer exchange coupling (see e.g. the paper of Bruno and Chappert ${ }^{11}$ :

$$
R^{2} \Delta \operatorname{LDOS}_{\text {vacuum }}(\varepsilon) \propto \frac{1}{\left|\frac{\mathrm{d}^{2} E}{\mathrm{~d} k_{x}^{2}} \cdot \frac{\mathrm{d}^{2} E}{\mathrm{~d} k_{y}^{2}}\right|}
$$

The denominator of this equation is a measure of the curvature of the constant energy surface, i.e., the shape of the constant energy surface affects the propagation of the electrons. Additionally, one can show that the electronic waves are directed parallel to $R$ by the group velocities. Since, experimentally states at the Fermi energy $\left(E_{\mathrm{F}}\right)$ are probed, the constant energy surface corresponds to the Fermi surface. Thus, the theoretically and experimentally observed charge density oscillations depend strongly on the shape of the Fermi surface: a small value of the curvature means that the Fermi surface has a flat region leading to large values of the LDOS and to strong focusing of intensity in this space region determined by the group velocity. In a previous report, ${ }^{12}$ we presented the DFT Fermi surface of $\mathrm{Cu}$ that is oriented with the (111)-neck direction normal to the drawing plane. On the Fermi surface we represented the denominator of the right hand side of Equation 2 with colors. The shape of the low values of this denominator, corresponding to the flat regions of the Fermi surface, is found to be rather triangular along the (111) direction in accordance with our simulations of the induced charge oscillations. One can understand the origin of the flat region seen within the triangle in Figure 2, which is induced by the neck of the $\mathrm{Cu}$ Fermi surface along the (111) direction that does not allow electrons to propagate.

The strong directionality of electron propagation even for a simple metal such as $\mathrm{Cu}$, has consequences in many fields. Once the shape of the propagator $G$ is known, either by STM-investigation or calculations based on DFT many additional effects can be predicted. For example, the spatially anisotropic characteristics should also be equally present in the RKKY interactions between magnetic impurities. Indeed, we have shown recently ${ }^{14}$ in connection with another experiment, that the interaction between Co adatoms on the $\mathrm{Pt}(111)$ surface is very anisotropic, which is obviously induced by the same physics discussed in this paper, i.e., the anisotropic shape of the Fermi surface.
2.2. Spin-Orbit Coupling: The Role of DzyaloshinskiiMoriya Interactions in Shaping Magnetic Domain Walls in Nanostructures

Whereas in a bulk material magnetic structure can be stabilized on the basis of spin-spin interactions alone, in lower dimensions SOC effects become decisive. They are described in the Dirac theory of the electron by a term in the Hamiltonian that has the form:

$$
\frac{\mu_{\mathrm{B}}}{2 c} \mathbf{\sigma}(\mathbf{E}(\mathbf{r}) \times \mathbf{p})
$$

where $\mathbf{p}$ is the momentum of the electron with spin $\boldsymbol{\sigma}$ moving in the electric field, $\mathrm{E}$, and $\mu_{\mathrm{B}}$ is the Bohr magneton. Despite its relatively small amplitude, the SOC term is responsible for a large class of important properties, such as the magnetic anisotropy that determines the magnetization orientation, or the antisymmetric exchange or DzayloshinskiiMoriya Interactions (DMIs).

While the magnetic anisotropy described above can be seen as a local, atomic quantity, SOC can also modify the interaction of spins on neighboring atoms. Besides the usual Heisenberg-type term, $J_{i j}\left(\mathbf{S}_{i} \cdot \mathbf{S}_{j}\right)$, in the presence of SOC an interaction of the spins of the form $\mathbf{D}_{i j} \cdot\left(\mathbf{S}_{i} \times \mathbf{S}_{j}\right)$ can come into play. The appearance of this interaction depends on certain symmetry conditions, which are seldom met in bulk metals but are always present at surfaces. Therefore, in magnetic nanostructures DMI is not an exotic phenomenon, although it has been mainly overlooked in the last decades. Our recent findings on ultrathin magnetic films clearly support this view. ${ }^{17-19}$

In the introduction, we mentioned that exchange interactions and SOC effects, i.e., the magnetic anisotropy, determine to a large extent the magnetic properties of low-dimensional magnetic systems. For a proper description of SOC effects, the DFT Hamiltonian has to contain a term such as Equation 3 or the full Dirac equation. For our calculations, we use Equation 3 with scalar-relativistic effects in a vector-DFT implementation of the full-potential linearized augmented plane-wave method. ${ }^{20}$ This allows us to extract both the scalar, Heisenberg-type interactions, $J$, and the magnetic anisotropy from total energy calculations. We note here, that in low-dimensional systems the latter quantity is mainly determined by SOC, while the dipole-dipole interaction adds only a minor contribution. This part can be evaluated via the classical dipole-dipole term once the magnetic moments have been determined from the DFT calculation. The SOC contribution is extracted from energy differences between two configurations with different magnetization directions.

A nice example, where the interplay of exchange interaction and magnetic anisotropy can be studied, is a magnetic domain wall, i.e., the region between two adjacent magnetic domains where the magnetization changes orientation (Figure 3). In out-of-plane magnetized ferromagnets, magnetic domains are formed either due to long-range dipolar interactions, which favor an antiferromagnetic (AFM) alignment of domains, or due to external magnetic fields that are used to write domain patterns in a material. For a high storage density of a magnetic data storage medium narrow domain walls are preferable. In a simple model, the domain wall 


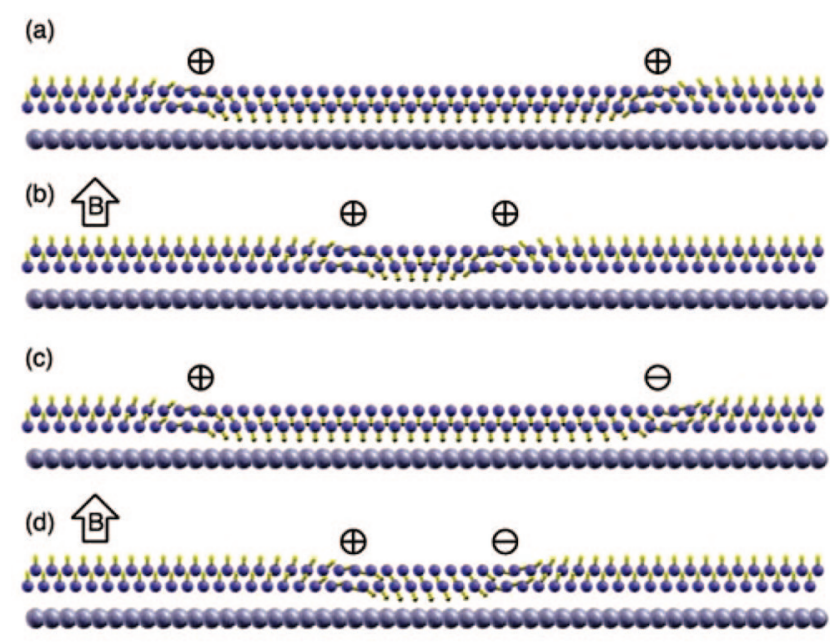

Figure 3. a) Magnetic domain of a thin film bounded by two Néeltype domain walls of the same chirality. b) If a magnetic field is applied, the domain with opposite orientation to the B-field shrinks and the domain walls move towards each other. Nevertheless, there is no magnetic torque in the center of the domain that could destabilize this magnetic entity. c) The same situation as in a), but now with domain walls of opposite chirality. d) If a magnetic field is applied, a torque acts throughout the domain and it will finally disappear, annihilating the $(+)$ and $(-)$ domain wall.

width, $w$, is given by $w=2(J / K)^{1 / 2}$, i.e., it is a compromise between the spin stiffness, $J$, that characterizes the energy cost to tilt two neighboring spins by an infinitesimally small angle, and the magnetic anisotropy, $K$, that describes the energy cost to rotate a spin away from the easy axis, in our example from the out-of-plane direction. Both quantities can be calculated reliably using DFT. ${ }^{19,} 20$

Next to its width, another interesting aspect in domain walls is its orientation: if two domains have to be formed, will they be separated by a wall that runs along a certain inplane direction and, if yes, which one? Here, the directional dependence of the spin-stiffness comes into play. As shown above, a considerable anisotropy in the exchange interaction can be induced by Fermi surface effects. Experimentally, two monolayer (ML) Fe on W(110) show an out-of-plane anisotropy and a clear preference for domain walls running along the [110] direction. Since this preference was not found in the calculations of the spin stiffness, other interactions that can account for this behavior have to be included in the model. The consequences of the DMI for magnetic domain walls are quite fundamental: First, it can determine the type of the domain wall. As we move along the $x$-direction, the magnetization can rotate either in the $(z, x)$ - or in the $(z, y)$-plane. The first case is called Néel-type wall, the second Bloch-type wall. Additionally, the DMI can determine the sense of rotation in these planes, clockwise or anticlockwise. For the symmetry of the studied problem this is only possible in Néel-type walls. Finally, since DMI prefers Néel-type walls with a certain sense of rotation, the magnetic anisotropy is now indirectly coupled to the direction of the domain wall and the DMI can also influence this property. As it turns out, in the case of Fe double-layers on W(110) this is indeed the case and the experimental findings can be explained from relativistic, noncollinear DFT calculations. ${ }^{19}$ It should be emphasized that these effects stem from a unique interplay of the broken symmetry at the surface (generally also found for chains or nanostructures on surfaces) and a large SOC. The latter effect can be traced back to the large atomic number of the substrate $(Z=74)$, thus these effects can be expected also on other frequently used materials such as gold or platinum. ${ }^{21}$

As a consequence of the DMI all domain walls have a certain rotational sense (sometimes called chirality) in the $\mathrm{Fe} / \mathrm{W}(110)$ system, as indicated in Figure 3a. Note, that this homochirality has also important implications for the stability of these domain walls with respect to external perturbations, e.g., an applied magnetic field B. As B increases, some domains will expand and others will shrink, causing a movement of the domain walls. The energy in the shrinking wall rises, as the structure gets increasingly non-collinear (Figure $3 b$ ). In a continuum model one would say that energy piles up when the two domain walls are squeezed together, in this way protecting the middle domain from annihilation. On the other hand, if the domain walls are of opposite chirality, as shown in Figure 3c, an applied magnetic field will be able to shrink and finally eliminate the oppositely oriented domain without this huge energy barrier (Figure 3d). Interestingly, experiments on the Fe/W(110) system using spinpolarized STM reported the existence of residual domains of a few nanometers that were found to be stable in magnetic fields. ${ }^{22}$ Our calculations identify the source of the homochirality of domain walls in this material, that leads to a kind of topological protection of domains in external fields.

Similar mechanisms can be exploited to stabilize also twodimensional magnetic structures, topological solitons called skyrmions. Both, domain walls and solitons are currently discussed as novel concepts for data storage devices.

\subsection{Spin-Orbit Coupling: Electrical Manipulation of Mag- netic Anisotropy in Metal-Organic Complexes}

On a more local scale, SOC gives rise to magnetocrystalline anisotropy, which determines the energy barriers that stabilize the magnetic moments against quantum tunneling and thermal fluctuations. These effects have been studied in detail in metallic nanostructures. However, organic molecular magnets are also very promising candidates as working units for spintronics. ${ }^{2-4}$ From the technological functionality point of view, besides a small size, they are required to have a strong ferromagnetic (FM) coupling of local spin moments and large values of MAE. Because of the reduced dimensions, nanometer-sized magnetic elements have to exhibit huge values of magnetic anisotropy per atom to resist thermal fluctuations of the magnetization direction. Moreover, controlling the magnetization direction, electronic and transport properties of such elements has to be local, avoiding, if possible, the use of external magnetic fields. A simple way to control the magnetization direction in organic magnetic molecules, common for the biomolecular world, is an oxidationreduction reaction. The transfer of electrons in or out of the molecule, the heart of an oxidation-reduction process, normally modifies not only the electronic properties of the molecule, but can also influence its magnetism drastically. In a previous report ${ }^{23}$ we demonstrated that for certain types of magnetic organic molecules the transfer of electrons in or out 


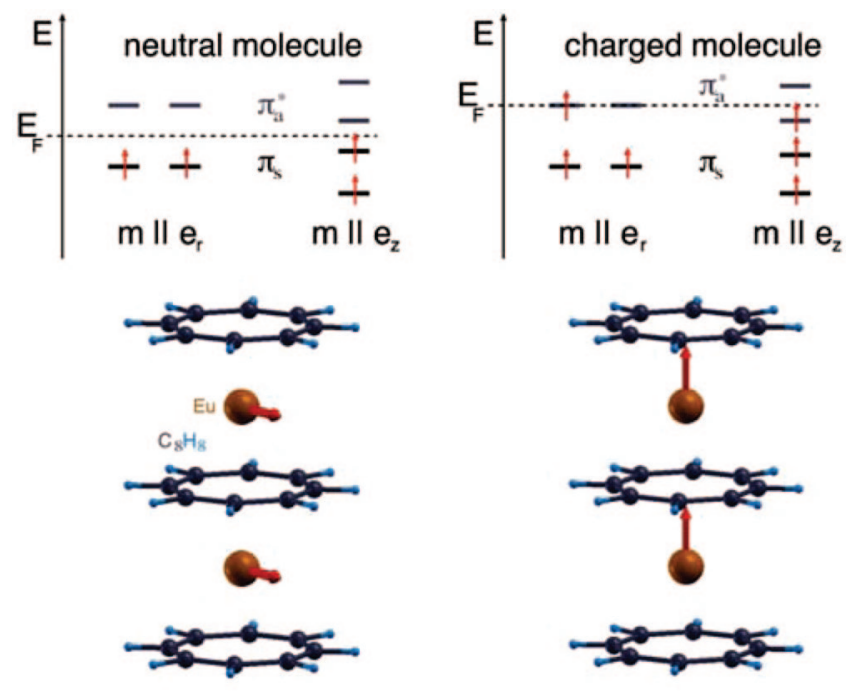

Figure 4. Highest occupied and lowest unoccupied levels in an $\mathrm{Eu}_{2}\left(\mathrm{C}_{8} \mathrm{H}_{8}\right)_{3}$ molecule in neutral (left) or singly charged state (right). If the magnetization is along the molecule axis $\left(m \| e_{z}\right)$, the $\pi$-levels split due to SOC. When $E_{\mathrm{F}}$ falls into this gap, a stabilization of the corresponding magnetization direction can be expected. Therefore, for a neutral molecule the easy axis is in radial $\left(e_{r}\right)$ direction, for the charged species in $z$-direction, as indicated in the lower part of the figure. Copyright (C) 2008 American Physical Society. Adapted with permission. ${ }^{23}$

of the molecule results in a drastic change in the magnetization direction, preserving the large spin due to a strong magnetic coupling between the metal ions and sizable magnetic anisotropies due to SOC. A dramatic example is the organometallic $\mathrm{Eu}_{2}\left(\mathrm{C}_{8} \mathrm{H}_{8}\right)_{3}$ molecule that can be regarded as a double-sandwich of Eu ions between $\mathrm{C}_{8} \mathrm{H}_{8}$ rings (Figure 4). The magnetic moments of the two $\mathrm{Eu}$ are coupled ferromagnetically and can be oriented either along the molecule axis $(z)$ or radial (r) (left and right in Figure 4). In DFT calculations it is found that the easy axis of this molecule is in $r$-direction, with an anisotropy energy of $2.68 \mathrm{meV}$ (energy difference to the $z$-direction). ${ }^{23}$ Interestingly, charging of the molecule completely alters its magnetic properties, leading to a magnetization along the molecule axis both for the anion and the cation. Our calculations confirm that all the $\mathrm{Eu}_{2}\left(\mathrm{C}_{8} \mathrm{H}_{8}\right)_{3}$ ionic species (with charge states $-2,-1,0,+1,+2$ ) have a FM ground state, with the total spin moment increasing linearly with the number of additional electrons: from $10 \mu_{\mathrm{B}}$ for $\mathrm{Eu}_{2}\left(\mathrm{C}_{8} \mathrm{H}_{8}\right)_{3}{ }^{+2}$ cation up to $14 \mu_{\mathrm{B}}$ for the $\mathrm{Eu}_{2}\left(\mathrm{C}_{8} \mathrm{H}_{8}\right)_{3}{ }^{-2}$ anion. Because of symmetry, near $E_{\mathrm{F}}$ two types of orbital arise as a result of the interaction between the $\mathrm{C}_{8} \mathrm{H}_{8}$ rings: symmetric $\left(\pi_{\mathrm{s}}\right)$ and antibonding antisymmetric $\left(\pi^{*}{ }_{a}\right)$. In the neutral compound, due to hybridization of the spin up $(\uparrow) \pi$ orbitals with the 4 f orbitals, the $\pi_{\mathrm{s}}$ orbitals are pushed to lower energies than the $\uparrow \pi_{\mathrm{a}}$ states which are pushed above $E_{\mathrm{F}}$ and become empty. This leads to a net magnetic moment of $2 \mu_{\mathrm{B}}$ carried by the $\Pi$ orbitals. The stabilization of a specific magnetization direction is a consequence of the modification of the gap between the highest occupied and lowest unoccupied levels by SOC, as shown in Figure 4. The position of this gap is in turn affected by charging: increasing negative charge of the $\uparrow \pi$ states are progressively filled, leading to different values of the SOC energy and orbital moments for different magnetization directions through $\pi-f$ hybridization. ${ }^{23}$
Such a study shows the possibility to manipulate the magnetic properties of organometallic molecules by electrical means. This may be achieved by subjecting the molecules to an electric potential, but also by chemical means. For practical purposes, however, organometallic molecules need to be interfaced with a solid substrate, which, especially in the case of metals, requires a thorough characterization of charge transfer and hybridization effects. These may affect the charge and spin of metal ions directly, as shown in Section 3.1 for adatoms deposited on metals as well as the strength of the ligand field surrounding the magnetic ions, as discussed in Section 3.2.

\section{Magnetic Atoms on Metallic and Nonmagnetic Substrates: Electron Hybridization Versus Correlation Effects}

Magnetic atoms on surfaces represent the ultimate limit of monodisperse magnetic particles. ${ }^{24}$ Although individual atoms generally present paramagnetic behavior, their investigation provides clues to fundamental and practical issues in magnetism, such as the survival of local moments in nanoparticles and thin films deposited on nonmagnetic supports, ${ }^{25,} 26$ the onset of magnetic anisotropy, ${ }^{6}, 27,28$ and the tendency to display either classical or quantum behavior. ${ }^{13,}$ 29, 30 Moreover, as shown in the previous sections, magnetic atoms can be used as a probe of Fermi surfaces 12 and substrate-mediated magnetic interactions. ${ }^{14}$

Narrow quasi-localized d-states play an important role in the magnetic and electronic behavior of low-dimensional transition-metal systems, including surfaces and nanoparticles. ${ }^{31}$ The case of individual magnetic impurities is highly nontrivial and may be considered as a benchmark for understanding electron correlation in transition metal systems. The established theory of atomic multiplet structure, which started from the seminal works of Racah, ${ }^{32}$ describes with great accuracy the magnetic moments of transition metal ions. ${ }^{33}$ The main mechanism of energy level formation in this case is related to the strong Coulomb interaction among 3d-electrons. The factor determining whether an atomic multiplet structure should form, or if energy bands are to be expected, is the competition between the Coulomb energy and the kinetic energy associated with electrons hopping from site to site in the lattice (Figure 5). For moderate hybridization between impurity and host electron states the Anderson model describes well the formation of a magnetic moment and the many-body spinflip processes may lead to the Kondo effect, ${ }^{34}$ which can be directly visualized by scanning tunneling spectroscopy. ${ }^{35,} 36$ When nanosized materials approach the atomic limit one may hence expect that conventional theoretical models fail, and the question is how to describe these systems appropriately.

To study the onset and progression of $\mathrm{d}$-sp hybridization for magnetic impurities on metal substrates we have employed STM and high-resolution photoemission spectroscopy. Fe atoms deposited on alkali metal films represent a useful model case, ${ }^{37}$ where, depending on the atomic volume of the alkali ions, the surface electron density can be decreased or increased. We find the hybridization of the Fe dstates to change drastically from being very weak for Cs to much stronger for Li. Figure 6a shows photoemission spectra for $0.01 \mathrm{ML}$ Fe atoms on Cs, K, Na, and Li hosts. From Cs 
Figure 5. Spectral function of a many-body $d$-electron impurity and host conduction electrons. Depending on the local Coulomb interaction and hybridization strength different behaviors are expected: localized impurity limit (bottom); intermediate coupling (middle); strong hybridization limit (top). Letters indicate multiplet $(\mathrm{M})$, quasi-particle resonance $(\mathrm{QP})$, lower and upper Hubbard bands (LHB, UHB) spectral features. Copyright (C) 2010 American Physical Society. Adapted with permission. ${ }^{37}$

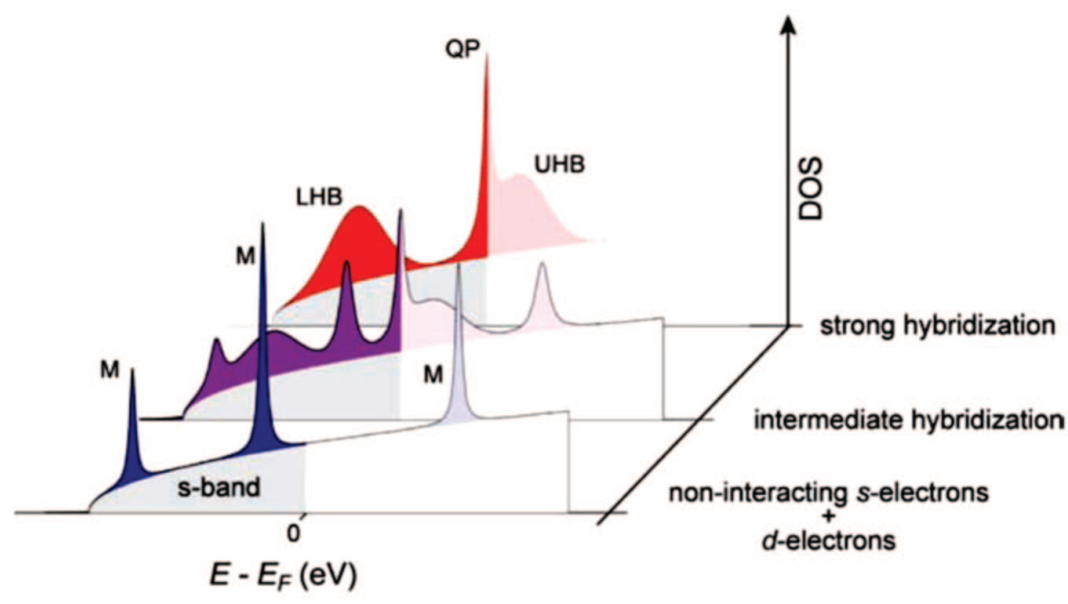

to $\mathrm{K}$ only a reduction of intensity of the multiplet features is observed accompanied by a moderate energy broadening. On $\mathrm{Na}$ and $\mathrm{Li}$, however, the spectra change qualitatively, indicating the onset and progression of $\mathrm{d}$-sp electron hybridization. For $\mathrm{Fe}$ on $\mathrm{Li}$ the spectrum presents a renormalized quasi-particle resonance near $E_{\mathrm{F}}$ and lower Hubbard bands around $-2 \mathrm{eV}$. In the Anderson model, the quasi-particle resonance peak near $E_{\mathrm{F}}$ is identified with the Kondo effect, representing low-energy excitations that involve the spin degrees of freedom of the impurity and conduction electrons. In this case, STM conductance spectra reveal an electronic resonance centered about $E_{\mathrm{F}}$ that is the signature of a manybody Kondo state 24,38 with a characteristic temperature $T_{K}$ $=96 \mathrm{~K}$ (Figure 6b,c). General ab initio theories of localizedto-itinerant electron behavior that go beyond DFT, such as the recently developed first-principles dynamical mean field theory, can be put to the test in order to improve our description of magnetism in solid state systems. ${ }^{37}$

Transition-metal substrates differ from free-electron-like metal hosts not only due to their larger conduction electron density, but also for the presence of unfilled d-states crossing $E_{\mathrm{F}}$, which heavily affect most of their magnetic properties (e.g., the susceptibility and magnetoresistance) as well as the nonmagnetic ones (e.g., cohesion, diffusion barriers, catalytic activity, etc.). As an example of a strongly interacting substrate we present data for isolated Co impurities on the (111) surface of Pt. 6, 24 X-ray absorption spectra (XAS) of Co/ $\mathrm{Pt}(111)$ differ from those of Co impurities deposited on freeelectron metals, ${ }^{25}$ as shown in Figure 7a. The narrow XAS multiplet structure observed for $\mathrm{Co} / \mathrm{K}$ indicates that the $\mathrm{Co}$ ground state has a strongly localized character, specifically a $\mathrm{d}^{8}$ configuration with atomic-like orbital $\left(m_{\mathrm{L}}\right)$ and spin $\left(m_{\mathrm{S}}\right)$ magnetic moments as determined by Hund's rules. This is in agreement with the trend observed for Fe impurities on alkali metal films. On Pt, on the other hand, the impurity $3 \mathrm{~d}$ states hybridize strongly with both the $5 \mathrm{~d}$ and 6 s-states of the substrate. Electron delocalization leads to a reduction of intra-atomic correlation effects, causing a substantial decrease of $m_{\mathrm{S}}$ and $m_{\mathrm{L}}$ compared to the case of $\mathrm{Co} / \mathrm{K}$. As shown both by experiments ${ }^{6,27}$ and ab initio calculations, ${ }^{39-41}$ this tendency increases gradually from impurities to larger nanoparticles and is governed by lateral adatom-adatom interactions as well as substrate-adatom hybridization.

Given the pronounced anisotropic spatial extension of the $d$-orbitals, the admixture of impurity and substrate $d$-states may lead to unequal filling of wave functions with different symmetry, and hence to a strong anisotropy of the orbital magnetization. As the SOC between $m_{\mathrm{S}}$ and $m_{\mathrm{L}}$ is taken into account, a strong magnetic anisotropy of the overall magnetization is expected, as discussed also in Section 2 of this work. For $\mathrm{Co} / \mathrm{Pt}$, the magnitude of the $\mathrm{XMCD}$ signal relative to the XAS intensity measured for $B=7 \mathrm{~T}$ applied out-ofplane and close to in-plane differs by more than $60 \%$, revealing the presence of extraordinary magnetic anisotropy for the Co adatoms in contact with the $\mathrm{Pt}(111)$ substrate (Figure $7 \mathrm{~b})$. The magneto-crystalline anisotropy energy can be determined by measuring the field dependence of the XMCD minimum at the $L_{3}$ edge along different directions with respect to the substrate normal, as shown in Figure $7 \mathrm{~b}$. A fit of the magnetization shows that the MAE of single Co atoms on $\mathrm{Pt}$ exceeds $9 \mathrm{meV}$ per atom and decreases very fast by increasing the average lateral coordination of Co adatoms,. These
Figure 6. a) Host-dependent Fe impurity photoemission spectra on free-electron like substrates. The data represent the impurity photoemission intensity after subtraction of the alkali background. The left scale reports the host free-electron density of Cs to Li. b) Spatiallyresolved Kondo resonances measured by STM corresponding to individual Fe impurities deposited on a 3.8 monolayer-thick Li film on $\mathrm{Cu}(100) . T=5 \mathrm{~K}$, set point $\mathrm{V}$ $=7 \mathrm{mV}, \mathrm{I}=0.1 \mathrm{nA}$, image size $100 \times 100 \AA^{2}$. c) Conductance spectrum measured on top of a Fe adatom (set point $\mathrm{V}=-100 \mathrm{mV}, \mathrm{I}=0.5 \mathrm{nA}$ ). The thick solid line is a fit to the data according to a Fano line-shape. Copyright (C) 2010 American Physical Society. Adapted with permission. ${ }^{37}$ (a)

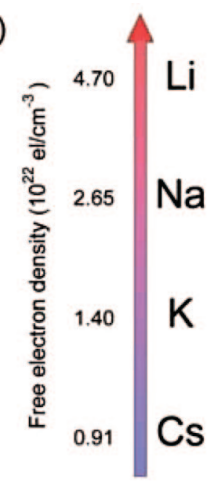

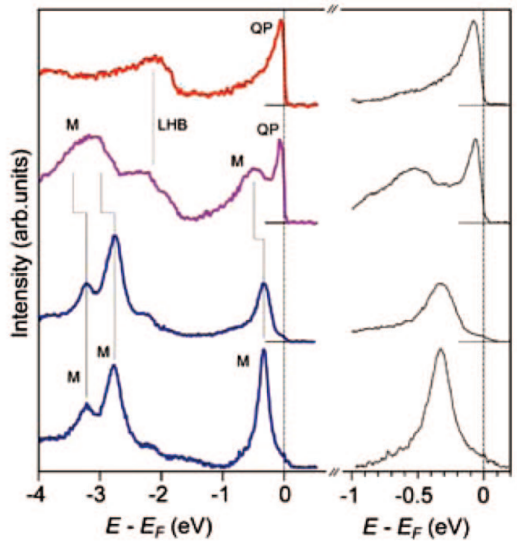

(b)

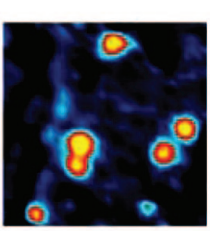

(c)

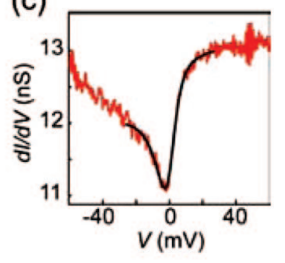


Figure 7. a) Experimental and theoretical $X$-ray absorption spectra of Co impurities in the $L_{3,2}$ edge region. The experimental spectra were recorded as the sum of the absorption intensity in total electron yield for parallel $\left(I^{+}\right)$and antiparallel $\left(I^{-}\right)$alignment of the photon helicity with respect to the magnetic field $B=$ $7 \mathrm{~T}$ at an angle $\theta=0^{\circ}$ relative to the surface normal. The temperature of the $\mathrm{Pt}(111)$ and $\mathrm{K}$ substrates was 6 and $10 \mathrm{~K}$, respectively. 6,25 The background absorption intensity [see spectra in (b)] has been subtracted from the Co/Pt(111) signal for comparison with the calculated XAS. The theoretical spectra were calculated using an atomic multiplet model ${ }^{42}$ for $\mathrm{d}^{8}$ and $\mathrm{d}^{7}$ configurations. b) XAS and XMCD of Co impurities on $\mathrm{Pt}(111)$ showing the strong anisotropy of the dichroic intensity as the magnetic field and photon helicity are directed out-of-plane $\left(\theta=0^{\circ}\right)$ and close to in-plane $\left(\theta=70^{\circ}\right)$. The inset shows the intensity of the XMCD minimum as a function of applied field at $6 \mathrm{~K}$. Copyright (C) 2003 American Association for the Advancement of Science; and copyright (C) 2002 American Physical Society. Adapted with permission. ${ }^{6,25}$

measurements show that an increase by one to two orders of magnitude in magnetic anisotropy energy with respect to bulk or 2D films can be obtained by reducing the size of magnetic particles to a few tens of atoms or less on suitable substrates. ${ }^{6,} 28$ While this holds on a per atom basis, it is obvious that the overall stability of the particle magnetization is governed by the sum of the atomic anisotropy energy contributions. As more atoms are assembled together to fabricate particles with a large total magnetic moment and a total MAE strong enough to stabilize FM behavior against thermal fluctuations, this gain is countered by the decrease of the MAE per atom with increasing size. The problem, however, can be circumvented by noting that the atomic coordination rather than the absolute particle size is the key parameter that governs the magnitude of the MAE, $m_{L^{\prime}}$ and $m_{S}$. Surface supported nanostructures where the shape and composition are tuned so as to control the coordination of the magnetic atoms and maximize useful interface effects, such as in coreshell 2D particles, ${ }^{43}$ nanowires, ${ }^{5,44-47}$ and metal-organic networks ${ }^{48}$ offer very interesting opportunities to exploit such effects, as discussed in the next Sections.

\section{Supramolecular Control of The Magnetic Anisot- ropy in Two-Dimensional High-Spin Fe Arrays at a Metal Interface}

As shown previously, individual atoms at surfaces are difficult to arrange in regular patterns; their magnetic properties are more often than not dominated by electronic hybridization with the substrate, which, as in the case of Kondo systems, may lead to a decrease or quench of their local magnetic moment. These problems can be overcome by embedding the magnetic atoms into a planar molecular framework. ${ }^{48}$ Note that, whereas the chemistry of metal-organic complexes is well-established, little is known on the electronic and magnetic properties of atomically-thin metal-organic grids interfaced with a metallic substrate or electrode. We have investigated two approaches to this problem, one relying on the deposition of macrocycle molecules such as metal-phthalocyanines ${ }^{49,50}$ and double-deckers ${ }^{51}$ on differ-

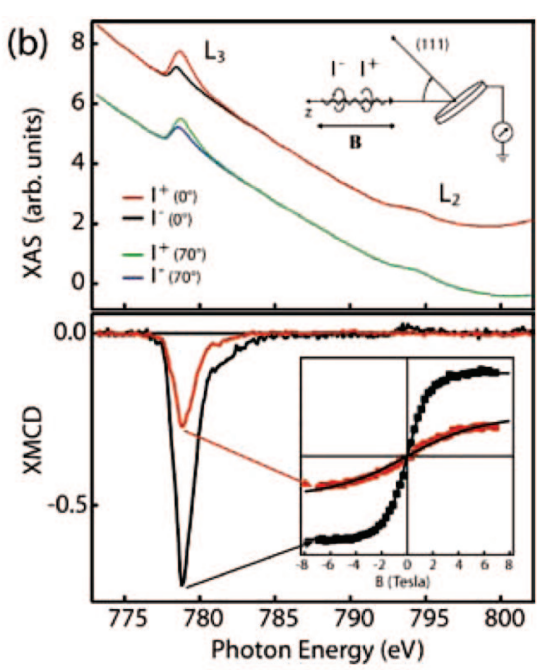

ent substrates, and the other on the supramolecular synthesis of mononuclear metal-organic networks on appropriate surfaces. ${ }^{48}$ We report here the results obtained using the latter approach, which allows for the fabrication of 2D metalorganic network structures by self-assembly that cannot be envisaged by standard synthesis methods.

The co-deposition of transition metal-ions and organic ligands on crystalline surfaces offers the potential to design supramolecular grids with programmable structural and chemical features, where the interaction with the substrate is used to stabilize a planar geometry. ${ }^{52,53} \mathrm{Fe}$ atoms coadsorbed with terephthalic acid (TPA) molecules on $\mathrm{Cu}(100)$ in ultrahigh-vacuum constitute a prototypical 2D heteroassembled system forming a variety of mono- and binuclear network structures, whose morphology is determined by the Fe:TPA stoichiometry, substrate symmetry, and annealing temperature. ${ }^{54}$ Figure 8 a shows a hard sphere model of the $\mathrm{Fe}(\mathrm{TPA})_{4}$ network investigated in this study, where each Fe atom is coordinated to four TPA molecules through Fe-carboxylate bonds, with the supramolecular Fe(TPA) ${ }_{4}$ units organized in a $(6 \times 6)$ unit cell with respect to the underlying $\mathrm{Cu}$ lattice. Weak hydrogen bonding interactions between the complexes favor long-range order extending over entire terraces of the substrate. The resultant superlattice of individual Fe atoms has perfect $15 \times 15 \AA^{2}$ periodicity. As will be shown below, lateral coordination with the molecular ligands yields unsaturated yet stable coordination bonds, which allow for the chemical modification of the electronic and magnetic properties of the Fe atoms independently from the substrate. Indeed, the Fe centers are chemically active, forming an array of open coordination sites that can bind additional axial ligands. This was proved by exposure to a moderate pressure of $\mathrm{O}_{2}\left(1 \times 10^{-8} \mathrm{mbar}\right)$ at room temperature, showing that adsorption of oxygen takes place close to Fe on-top positions, as evidenced by DFT calculations (Figure 8b) and STM (Figure 8c). An $\mathrm{O}_{2}$ dose larger than $200 \mathrm{~L}$ leads to saturation of the Fe sites, corresponding to a $\mathrm{O}_{2}-\mathrm{Fe}(\mathrm{TPA})_{4}$ network.

In order to probe the chemical bonds and magnetism of the Fe(TPA) $)_{4}$ layer, we performed XAS at the Fe L-edges and $\mathrm{O}$ K-edge. The intensity, lineshape, and polarization dependence of dipole-allowed $2 \mathrm{p} \rightarrow 3 \mathrm{~d}$ transitions offer information on the spin state, oxidation state, and ligand field of Fe ions in an element-selective way. Figure 9 shows the XAS 
Figure 8. Planar supramolecular layers of FeTPA complexes self-assembled on $\mathrm{Cu}(100)$. a) Ball and stick model of $\mathrm{Fe}(\mathrm{TPA})_{4}$ array. Blue dots indicate the position of Fe atoms, red $\mathrm{O}$, black $\mathrm{C}$, white $\mathrm{H}$. b) Perspective structure of the $\mathrm{O}_{2}-\mathrm{Fe}(\mathrm{TPA})_{4}$ array as calculated by DFT. The green halo represents the spin density. c) STM image of a $\mathrm{Fe}(\mathrm{TPA})_{4}$ monolayer grown on $\mathrm{Cu}(100)$. Selective uptake of $\mathrm{O}_{2}$ by Fe sites is indicated by a change of STM contrast (white spots). Lateral image size $84 \times 84 \AA^{2}$. Copyright (C) 2009 Nature Publishing Group. Adapted with permission. ${ }^{48}$
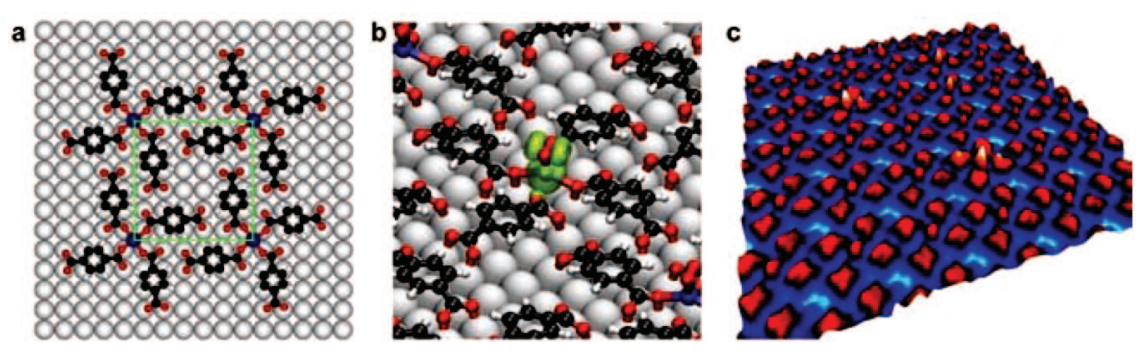
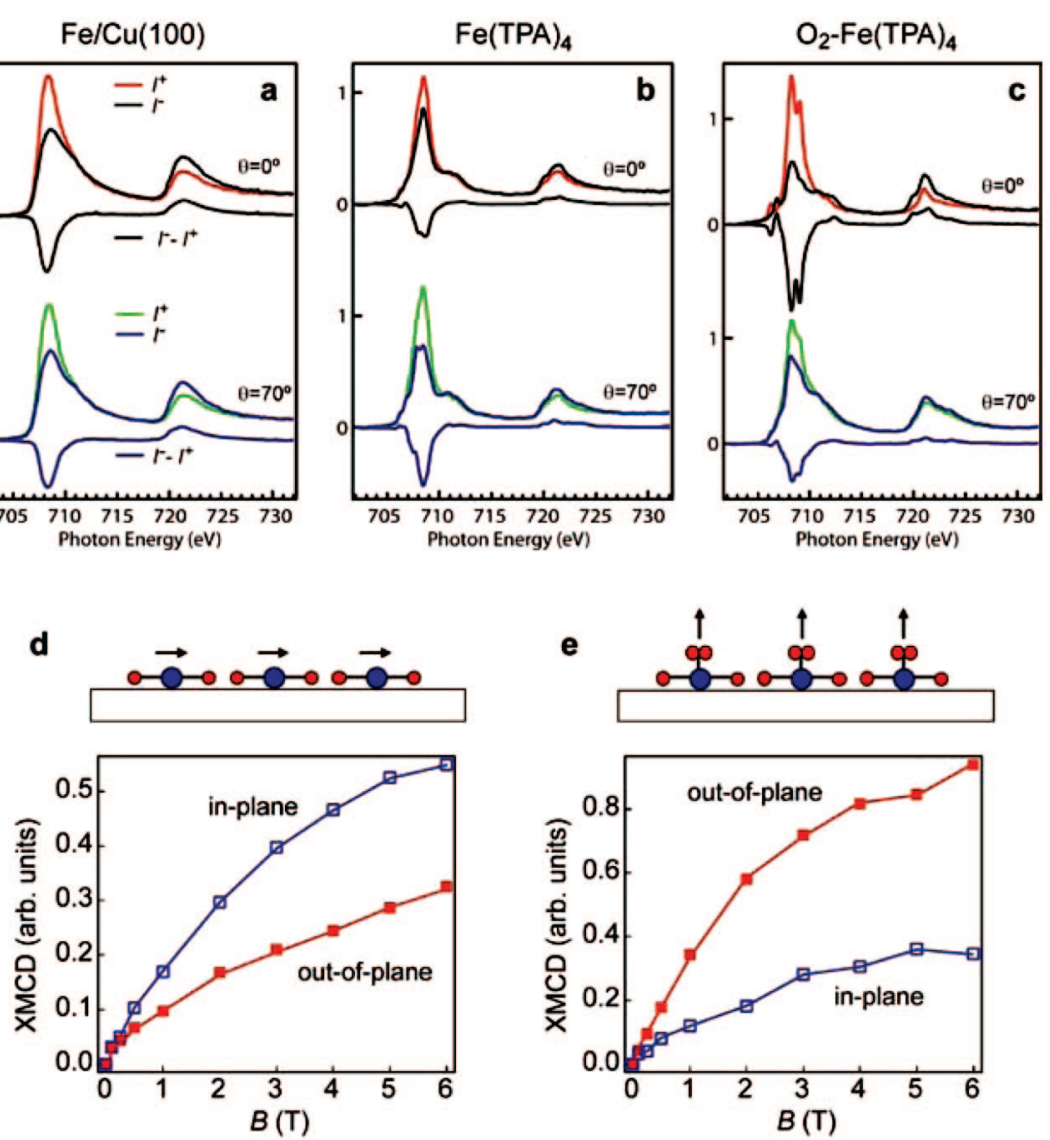

Figure 9. Top row: circularly polarized XAS of a) 0.025 monolayers $\mathrm{Fe} / \mathrm{Cu}(100)$, b) $\mathrm{Fe}(\mathrm{TPA})_{4}$, and c) $\mathrm{O}_{2}-\mathrm{Fe}(\mathrm{TPA})_{4}$ for parallel $\left(I^{+}\right)$and antiparallel $\left(I^{-}\right)$orientation of the photon helicity with field-induced magnetization. Spectra were recorded in the electron-yield mode at normal $\left(\theta=0^{\circ}\right)$ and grazing incidence $\left(\theta=70^{\circ}\right)$ at $T=8 \mathrm{~K}, B=6 \mathrm{~T}$. Bottom row: element-selective magnetization curves of the $\mathrm{Fe}$ centers of d) $\mathrm{Fe}(\mathrm{TPA})_{4}$ and e) $\mathrm{O}_{2}-\mathrm{Fe}(\mathrm{TPA})_{4}$ measured by XMCD at $T=8 \mathrm{~K}$ with the field applied out-ofplane $\left(\theta=0^{\circ}\right.$, full symbols) and close to the in-plane direction $\left(\theta=70^{\circ}\right.$, open symbols). The data points represent the Fe XMCD intensity integrated over the $L_{3}$ edge and normalized by the corresponding $L_{3}$ inaxis magnetization direction. Copyright (c) $2009 \mathrm{Na}$ ture Publishing Group. Adapted with permission. ${ }^{48}$

and XMCD spectra of a) 0.025 monolayers Fe deposited on $\mathrm{Cu}(100)$ at room temperature, equivalent to the Fe content in the supramolecular arrays, b) $\mathrm{Fe}(\mathrm{TPA})_{4}$, and c) $\mathrm{O}_{2}$-Fe(TPA) $)_{4}$. The XAS intensity, shown after $\mathrm{Cu}$ background subtraction, was measured at $8 \mathrm{~K}$ with magnetic field $B=6 \mathrm{~T}$ applied out-of-plane $\left(\theta=0^{\circ}\right)$ and at grazing incidence $\left(\theta=70^{\circ}\right)$ for parallel $\left(I^{+}\right)$and antiparallel $\left(I^{-}\right)$alignment of the photon helicity with B. More details about the measurements have been reported previously. ${ }^{48}$ The comparison of Figure $9 a, b$, and c reveals telltale differences in the XAS lineshape:

while $\mathrm{Fe} / \mathrm{Cu}(100)$ presents broad $L_{3}$ and $L_{2}$ peaks typical of a metal, pronounced narrowing of the spectral features and a well-defined multiplet fine structure is observed for the Fe-TPA complexes, which recall the spectra of $\mathrm{Fe}$ ions in bulk metal-organic compounds. ${ }^{55}$ These changes, related to ligand-induced modifications and localization of the Fe $d$-orbitals, indicate that coordination bonds have formed between the Fe centers and carboxylate ligands, with partial decoupling of Fe from the metal substrate. Such bonds, which involve Fe $3 \mathrm{~d}$ and $\mathrm{O} 2 \mathrm{p}$ states, explain the 4-fold coordination geometry as well as the thermal stability of this compounds. Interestingly, electron localization effects increase gradually from $\mathrm{Fe}(\mathrm{TPA})_{4}$ to $\mathrm{O}_{2}-$ $\mathrm{Fe}(\mathrm{TPA})_{4}$, so that additional ligands are found to favor electronic decoupling of the Fe atoms from the substrate.

XAS simulations using ligand field multiplet theory indicate that the ground state of Fe in both $\mathrm{Fe}(\mathrm{TPA})_{4}$ and $\mathrm{O}_{2}-$ $\mathrm{Fe}(\mathrm{TPA})_{4}$ complexes has almost pure $d^{6}$ character $\left(\mathrm{Fe}^{2+}\right)$, with maximum $14 \%$ and $15 \% d^{7}$ weight, respectively, depending on the hopping parameters assumed in the calculations. ${ }^{48}$ This result has important consequences as it confirms that the $\mathrm{Fe} d$-orbitals are interacting mainly with the ligands and proves that $\mathrm{Fe}(\mathrm{TPA})_{4}$ and $\mathrm{O}_{2}-\mathrm{Fe}(\mathrm{TPA})_{4}$ constitute highspin $\mathrm{Fe}^{2+}$ complexes. Moreover, $\mathrm{Fe}^{2+}$ is expected to favor large zero field splitting and anisotropic g-factors, i.e., strong magnetic anisotropy properties. Consistently with the geometry of the $\mathrm{O}$ ligands, we find that the crystal field splitting is close to square planar and square pyramidal for Fe(TPA) and $\mathrm{O}_{2}-\mathrm{Fe}(\mathrm{TPA})_{4}$, respectively, while the strong tetragonal distortion that characterizes $\mathrm{Fe}(\mathrm{TPA})_{4}$ reduces significantly in $\mathrm{O}_{2}-\mathrm{Fe}(\mathrm{TPA})_{4}$ due to the presence of the apical ligand. DFT calculations further show that the total number of Fe valence electrons changes from 6.67 to 6.33 following $\mathrm{O}_{2}$ chemisorption, but that the population of the $3 \mathrm{~d}$-states remains essentially stable, in agreement with the ligand field simulations. There are 0.8 excess electrons localized on the $\mathrm{O}_{2}$ ligand, 
whose largest fraction is supplied by the $\mathrm{Cu}$ substrate acting as a charge reservoir, a behavior that appears to be specific of surface-supported metal-organic systems. Notably, the formation of the supramolecular complexes is accompanied by a substantial increase of the Fe-substrate distance, calculated as $2.32 \AA$ for individual $\mathrm{Fe}$ atoms on $\mathrm{Cu}(100), 2.71 \AA$ in $\mathrm{Fe}(\mathrm{TPA})_{4}$, and $3.32 \AA$ in $\mathrm{O}_{2}-\mathrm{Fe}(\mathrm{TPA})_{4}$, showing alongside with XAS how the $\mathrm{Fe}-\mathrm{Cu}$ metal interactions progressively weaken with increasing number of ligands. ${ }^{48}$

The XMCD spectra reveal the presence of large local magnetic moments on $\mathrm{Fe}$, and in particular of increasing orbital moments going from $\mathrm{Fe} / \mathrm{Cu}(100)\left(0.18 \pm 0.03 \mu_{\mathrm{B}}\right)$, to $\mathrm{Fe}(\mathrm{TPA})_{4}\left(0.42 \pm 0.06 \mu_{\mathrm{B}}\right)$, and to $\mathrm{O}_{2}-\mathrm{Fe}(\mathrm{TPA})_{4}\left(0.55 \pm 0.07 \mu_{\mathrm{B}}\right)$, measured at $T=8 \mathrm{~K}$, with $B=6 \mathrm{~T}$ applied parallel to the easy magnetization direction. Most importantly, angle-dependent XMCD measurements show that the Fe magnetization is nearly isotropic on $\mathrm{Cu}(100)$, but becomes strongly anisotropic upon the formation of the metal-organic networks. Moreover, whereas the $\mathrm{Fe}(\mathrm{TPA})_{4}$ easy axis lies in-plane, $\mathrm{O}_{2}$ adsorption at the Fe sites further drives an abrupt spin reorientation transition, rotating the Fe easy axis out-of-plane (Figure 9d,e). The cause for the easy axis switch in supported metal-organic complexes is however different from that proposed for free molecules in Section 2.3, based on the control of the metal oxidation state. ${ }^{23}$ Our results show that only the ligand field is affected by $\mathrm{O}_{2}$ adsorption, while the formal Fe oxidation does not change. The axial $\mathrm{O}_{2}$ ligand induces a change of the Fe ground state from $\mathrm{A}_{1 \mathrm{~g}}$ to $\mathrm{E}_{\mathrm{g}}$ in $\mathrm{O}_{2}-$ $\mathrm{Fe}(\mathrm{TPA})_{4}$, as expected based on symmetry arguments. The $\mathrm{E}_{\mathrm{g}}$ term is an orbitally-degenerate doublet with nonzero orbital moment pointing along the principal symmetry direction, which explains the tendency of $\mathrm{O}_{2}-\mathrm{Fe}(\mathrm{TPA})_{4}$ to magnetize out-of-plane, together with its enhanced orbital moment compared to $\mathrm{Fe}(\mathrm{TPA})_{4}$.

Controlling magnetic anisotropy independently from the substrate is a key issue in the development of molecule-metal interfaces for magnetic applications, both at the single molecule ${ }^{56}$ and extended film level. ${ }^{57}$ With respect to bulk molecular crystals, the planar and open coordination structure of the self-assembled Fe array makes such a system extremely sensitive to ligand modifications, providing a handle to the preferred Fe spin orientation and enhanced chemical sensitivity of the magnetization. The capability to fabricate 2D arrays of monodisperse spin centers with nanometer spacing, and to understand and control their magnetic properties at the interface with a metal substrate, constitutes a basic step towards the exploitation of single spin phenomena in heterogeneous devices based on either a mono- or multilayer geometry.

\section{Complex Magnetic Phase in Submonolayer Fe Stripes on Pt(997)}

Concerning purely metallic systems, the assembly of magnetic atoms into particles of nanometer size and well-defined shape constitutes the primary route to the design of functional systems. It offers several degrees of freedom for tuning the properties of the single particle and of their ensemble. The magnetic properties of nanostructures are highly susceptible to changes in cluster size and geometry as well as their contact area to a supporting substrate. For the smallest clusters the magnetic properties usually strongly oscillate with every atom that is added or removed, a fact that is often expressed as "every atom counts." In the single atom limit we refer to the giant magnetic anisotropy effect of a single Co adatom situated on $\operatorname{Pt}(111)^{6}$ discussed in Section 3.2. In this ultimate limit the combination of the strongly reduced and anisotropic coordination of Co atoms and the large SOC constants of Pt leads to an extraordinary large out-of plane magnetic anisotropy energy of about $9 \mathrm{meV}$ per atom. Increasing the lateral degree of Co-Co coordination during formation of 2D Co-islands the magnetic easy axis remains perpendicular to the surface, however, the MAE, which strongly depends on the degree of coordination, is noticeably decreased.

The local atomic coordination is one important parameter influencing the magnetic properties of nanostructures. Another one is the chemical composition, determining element specific $d$-band filling effects which can dramatically alter the magnetic properties. In the following, fundamental magnetic properties of $\mathrm{Fe}$ and $\mathrm{Co}$ nanostructures on $\mathrm{Pt}(997)$ substrates are compared. Fe is interesting from a fundamental physics point of view since, compared to $\mathrm{Co}$, it has a stronger tendency to form complex magnetic phases like e.g. AFM and non-collinear magnetic ordering on heavy $4 \mathrm{~d}^{58}$ and $5 d^{59}$ transition metal substrates. The basic reason for this more rich behavior is that Fe lays at the borderline between the lighter $3 d$ AFM elements and the heavier ones, for which the direct exchange favors a FM coupling.

We will show that Fe nanostructures on $\mathrm{Pt}(997)$ are a perfect model system to study both Fe-Fe and Fe-Pt coordination effects at the same time, since depending on the conditions during preparation systems with different symmetry, dimension and local coordination are formed. The Pt substrate presents a regular sequence of terrace and single-atom steps and acts as a template for the growth of atomic chains and 2D clusters. The magnetic characterization of these systems was again accomplished by XMCD. STM was used to gain exact information about the quality of the growth of the nanostructures and their evolution with increasing $\mathrm{Fe}$ amount on the Pt surface. The STM images in Figure 10a are taken at $T=4 \mathrm{~K}$ after sample preparation at room temperature. At Fe coverages of about $x=0.1 \mathrm{ML}$ ( $1 \mathrm{ML}$ is defined as the number of a Fe atoms required to form a complete atomic layer on the Pt surface) the Fe atoms decorate the steps and form an array of well-ordered 1D chains of atoms along the [110] direction, as it was observed for $\mathrm{Co} / \mathrm{Pt}(997) .5,44$ The high degree of order of these 1D Fe structures leads to a pronounced peak in thermal energy atomic scattering (TEAS) at grazing incidence as shown in Figure 10b (a detailed discussion of the TEAS results was reported previously ${ }^{60}$ ). Beyond the monoatomic chain range, however, the order is lost and no further peaks in the TEAS are observed. Consistently with this observation, the STM images reveal a more and more corrugated step-flow growth with increasing coverages $0.13<x<1 \mathrm{ML}$. Moreover, on a larger scale it is evident from further investigation with STM at around 0.7 ML (not shown here) that a 2D equivalent to Stranski-Krastanov growth is observed, which leads to the formation of blocks. A careful statistical evaluation of the large scale STM images shows that $2 \mathrm{D}$ block formation occurs at about $75 \pm 5 \%$ of a Pt step edge. While the grazing incidence TEAS signal is sensitive to processes at the step edges, the non-grazing incidence provides information on the terrace morphology. 

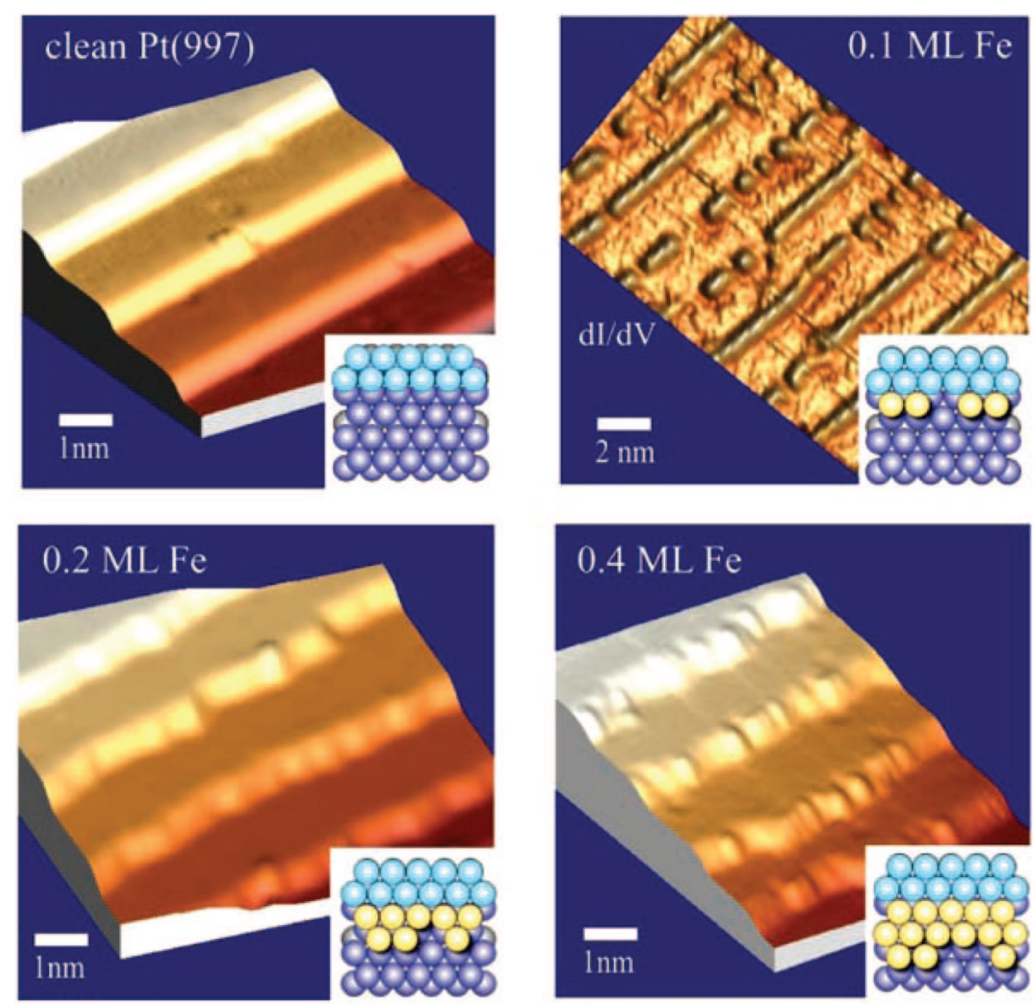

a)
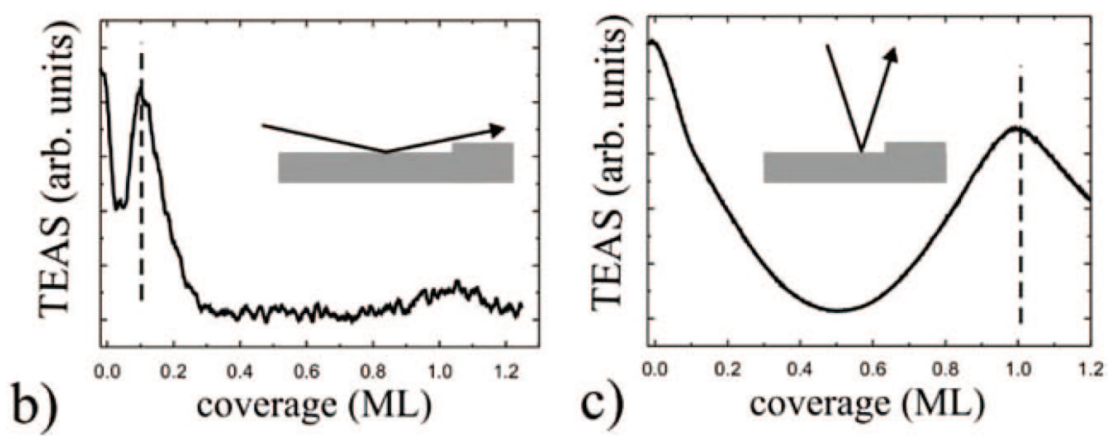

magnitude of the MAE, as summarized in Figure 11a. Co chains prefer to align their moments $43^{\circ}$ away from the surface normal, ${ }^{5}$ while the easy axis of $\mathrm{Fe}$ is almost in-plane, at $80^{\circ}$ from the surface normal. ${ }^{47}$

The distinct change in the magnetic easy axis direction for the two neighboring $3 d$ elements $\mathrm{Co}$ and $\mathrm{Fe}$ in the table of elements can be qualitatively reproduced using DFT calculations in the spin-density approximation. ${ }^{61}$ The ab initio approach models the system by a supercell, which contains 13 atoms (12 Pt plus one Co or Fe atom). As a general result, the calculations unambiguously show that the MAE is largely driven by the SOC constant of $\mathrm{Pt}$ and the axis along the wire is a hard axis. The MAE becomes very small when the SOC constant of $\mathrm{Pt}$ is set to zero. For the easy axis directions of $\mathrm{Co}$ and $\mathrm{Fe}$ chains these calculations predict angles of $23^{\circ}$ and $75^{\circ}$ with respect to the surface normal towards the step-edge, respectively, which is in good agreement with the measurements. It should be pointed out, however, that the value of the angle of the easy axis is susceptible to changes in the super cell size ${ }^{61}$ From the magnetization curves in Figure 11c, $\mathrm{d}$ there is evidence that the MAE is larger in the case of Co: at a temperature of $10 \mathrm{~K}$ the $\mathrm{Co}$ chains show a remanent behavior after saturation along the easy axis direction, while $\mathrm{Fe}$ still shows reversible superparamagnetic behavior. This finding reflects the larger MAE in $\mathrm{Co}$, which is sufficiently large to stabilize ferromagnetism for the chains at the temperature of $8 \mathrm{~K}$. This confirms the trend found for single impurities on $\mathrm{Pt}$ substrates where the magnetic anisotropy caused by the contact of $3 \mathrm{~d}$ metal with Pt is found to be considerably larger in the case of Co.

Figure 10. Growth behavior of Fe on $\mathrm{Pt}(997)$ at $T=300 \mathrm{~K}$. a) Typical STM images for coverages between $0<x<0.4 \mathrm{ML}$. The sketches in the insets model the geometry (Fe shown in yellow). TEAS at b) grazing and c) normal incidence measured perpendicular to the substrate steps. Copyright (c) 2009 American Physical Society. Adapted with permission 47

The peak appearing at exactly $1 \mathrm{ML}$ (Figure 10c) is consistent with the pseudomorphic growth of Fe on $\mathrm{Pt}(997)$ and shows the strong tendency of Fe to fully cover the Pt terraces before forming Fe double layers.

As discussed above, single adatoms and 1D chains form when deposition of small amounts of material is performed at low $(T=10 \mathrm{~K})$ and intermediate $(T=300 \mathrm{~K})$ temperatures, respectively. Comparing the results of Fe with earlier measurements on $\mathrm{Co}^{5,44}$ one finds that in both cases a low average lateral $3 d$ metal coordination leads to increased spin and orbital moments per atom compared to bulk, which are ferromagnetically coupled and show a superparamagnetic response to external magnetic fields. Differences in the magnetic properties of 1D Fe or Co chain geometries are visible in the orientation of the magnetic easy axis and in the
For 1D chains the magnetization curves of both Fe and Co can be fitted using a simple superparamagnetic model, ${ }^{5}$ which assumes a homogeneously polarized macrospin of $N$ atoms with enhanced $3 \mathrm{~d}$ moments per atom compared to bulk. Beyond the monochain coverage $(x>0.13 \mathrm{ML})$ a substantially different behavior is observed. For Fe on Pt(997) at intermediate coverages $x=0.2 \mathrm{ML}$ a low-spin phase (Figure 12a) is formed where the average effective spin moment per Fe atom is reduced by more than a factor of $4 .{ }^{47}$ The $0.1 \mathrm{ML}$ and 0.2 ML coverages correspond to approximately the single $1 \mathrm{D}$ chain $(0.13 \mathrm{ML})$ and the double-chain configuration (0.26 ML). The comparison between polar (dashed lines) and in-plane (full lines) magnetization curves in Figure 12b,c furthermore shows, in contrast to the single chain behavior, that at $x=0.2 \mathrm{ML}$ the difference between the in-plane and outof-plane magnetization curve vanishes. Since the magnetic easy axis direction changes only slightly, this degeneracy between the two directions is rather attributed to a softening of the average MAE. This is clearly shown in Figure 12e,f, by the measurement of the magnetization in a constant magnetic field of $1 \mathrm{~T}$ for different polar angles $\theta$. In both cases the 


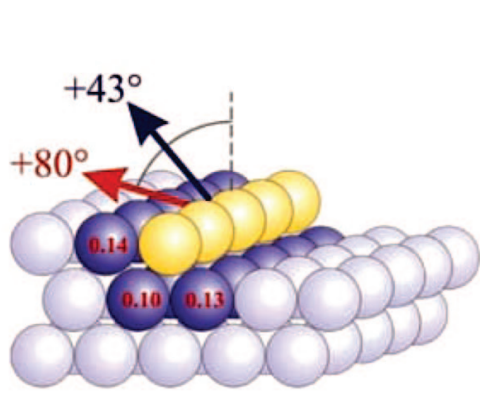

a)
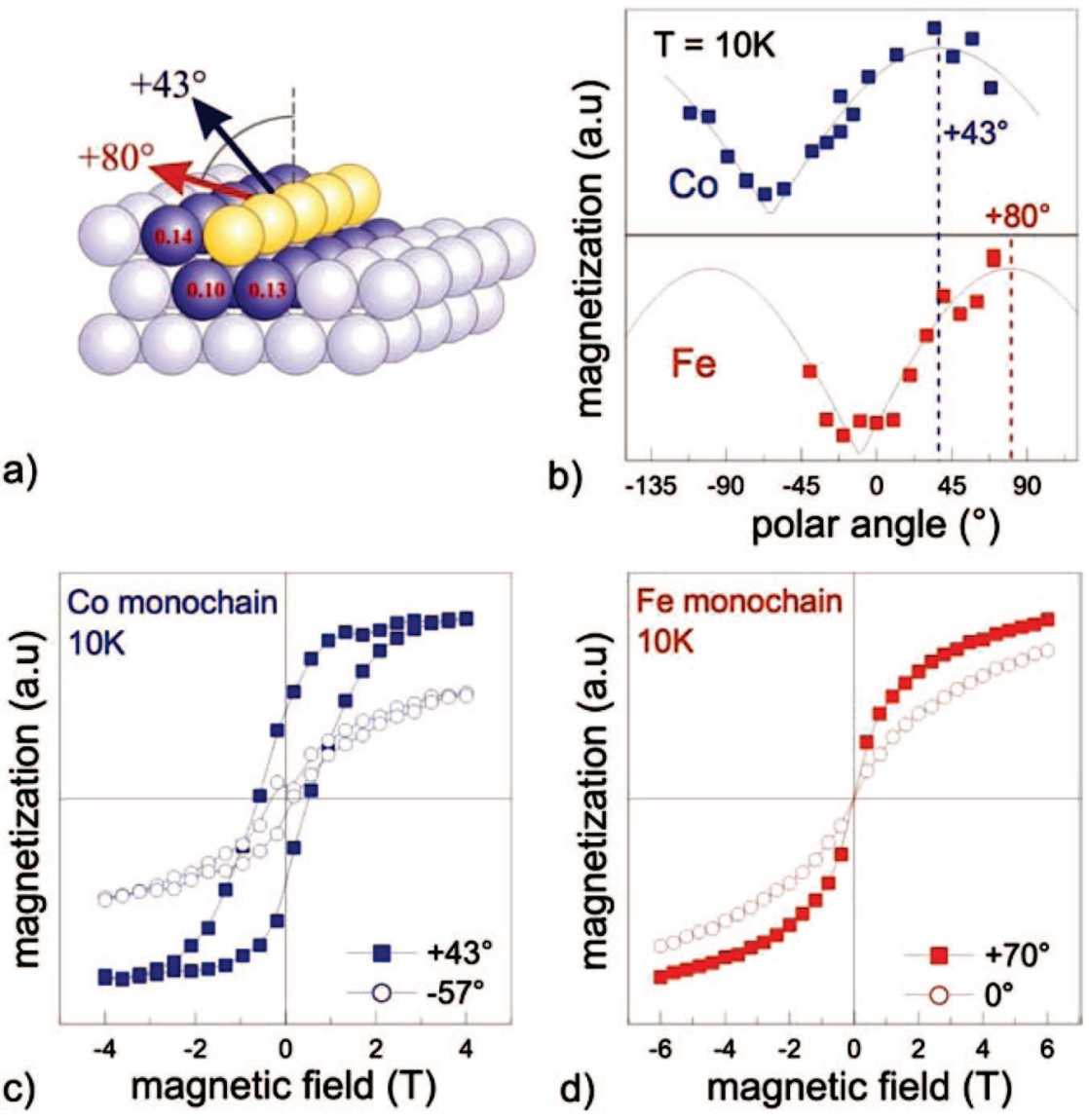

Figure 11. 1D Fe and Co chains grown on a Pt(997) template. a) The wires are aligned along the [111]-oriented step-edges between adjacent (111) terraces of platinum. b) Magnetization of $\mathrm{Co}$ and $\mathrm{Fe}$ at intermediate fields for different direction perpendicular to the step edges of $\mathrm{Pt}(997)$. c,d) Co and Fe magnetization curves taken close to the respective easy and hard axis directions. Copyright 2002, Nature Publishing Group; and copyright (C) 2009 American Physical Society. Adapted with permission. 5,47

minimum in the induced magnetization defining the hard axis direction is close to $\theta=0^{\circ}$. Also in Figure $12 \mathrm{f}$ the softening of the magnetic anisotropy energy is visible in the comparably weak angle dependence of the measured magnetization. At higher coverages (0.7 ML) the magnetic easy axis is fully rotated to the out-of-plane direction. This is evident from Figure 12g, where the remanent magnetization is plotted for different angles $\theta$. The hard axis is found close to the in-plane direction for higher coverages, in agreement with earlier MOKE measurements at higher temperatures $T=130$ K, where $1 \mathrm{ML}$ Fe on $\mathrm{Pt}(997)$ was shown to be out-of-plane magnetized. ${ }^{62}$ At the same time Figure 12a shows that the average moments per atom have gradually recovered with increasing Fe coverage.

The strong dependence of the average moment on the geometry in Fe nanostructures on $\mathrm{Pt}(997)$ systems points towards a complex compensated magnetic structure determined by a competition of FM coupling with AFM interactions, Dzyaloshinski-Moriya type interactions, or diverse on-site MAE for differently coordinated Fe atoms. Both the effects due to DMI and MAE can be greatly enhanced as they are relativistic in origin and profit from the large spin-orbit constant of $\mathrm{Pt}$. The complexity in the magnetism observed in $\mathrm{Fe} / \mathrm{Pt}(997)$ is reflected in the complex structure observed in the STM studies, where the rim of Fe stripes is shown to be strongly corrugated on the atomic scale (Figure 10b). This leads to a manifold of coordination states of $\mathrm{Fe}$, and thus a strong site-dependence of the magnetic properties can be expected. We can take the softening of the magnetic anisotropy at the critical coverage of $x=0.2 \mathrm{ML}$ as a strong indication for the presence of local variations in on-site Fe anisotropies. The reduction in the anisotropy is explained by a simple random anisotropy model (RAM) as proposed by Herzer. ${ }^{63}$ According to this model, the dominating exchange interaction forces the local moments to be collinear, however, due to the randomness in the easy axis directions the anisotropy averages to small values. This model can in principle be extended to more complex non-collinear structures like e.g. DMI-induced spiral configurations. For Fe/ $\mathrm{Pt}$ (997) we propose the presence of both FM and AFM coupling, depending on the local bonding configuration, leading to a small average magnetization and reduced anisotropies according to the RAM. Indeed, FM and AFM exchange couplings in free-standing 2D Fe triangles were predicted by theory depending on their electronic interaction. ${ }^{64}$ In contrast, perfect double chains on $\mathrm{Pt}(997)$ have a FM ground state according to DFT calculations, ${ }^{61}$ which again points towards the importance of a certain degree of disorder in the system. We also want to mention the possibility of local strain relaxation in the rim region leading to changes in the $\mathrm{Fe}-\mathrm{Fe}$ distances and interactions.

Thus, we ascribe the low magnetization phase found in $\mathrm{Fe} / \mathrm{Pt}(997)$ systems to a complex magnetic configuration in corrugated rim regions of Fe islands, leading to both FM and AFM coupling of Fe moments depending on local bonding configurations. The result of this work demonstrates the complexity of the magnetism in low-dimensional Fe metal structures on surfaces. In contrast to other Fe systems, such as well-ordered full monolayers of Fe on $\operatorname{Ir}(111)^{59}$ where joint $\mathrm{Fe}-\mathrm{Fe}$ and Fe-substrate interactions were shown to promote a long-range-ordered phase driven by DMI and AFM interactions, our work addresses the formation of complex Fe magnetism on a local scale in laterally confined nanostructures.

\section{Self-Assembled Superlattices of Non-Interacting Magnetic Nanoislands}

In Sections 4 and 5 we have described the self-assembly of magnetic units that are either 0D (atoms) or 1D (wires). Here, we go one step further to illustrate the fabrication of ordered arrays of 2D magnetic particles using a surface template that presents a uniform network of nucleation sites. Such a template is provided by the herringbone reconstruction typical of the (111) face of Au with the additional constraint provided by a slight miscut of the substrate. The 

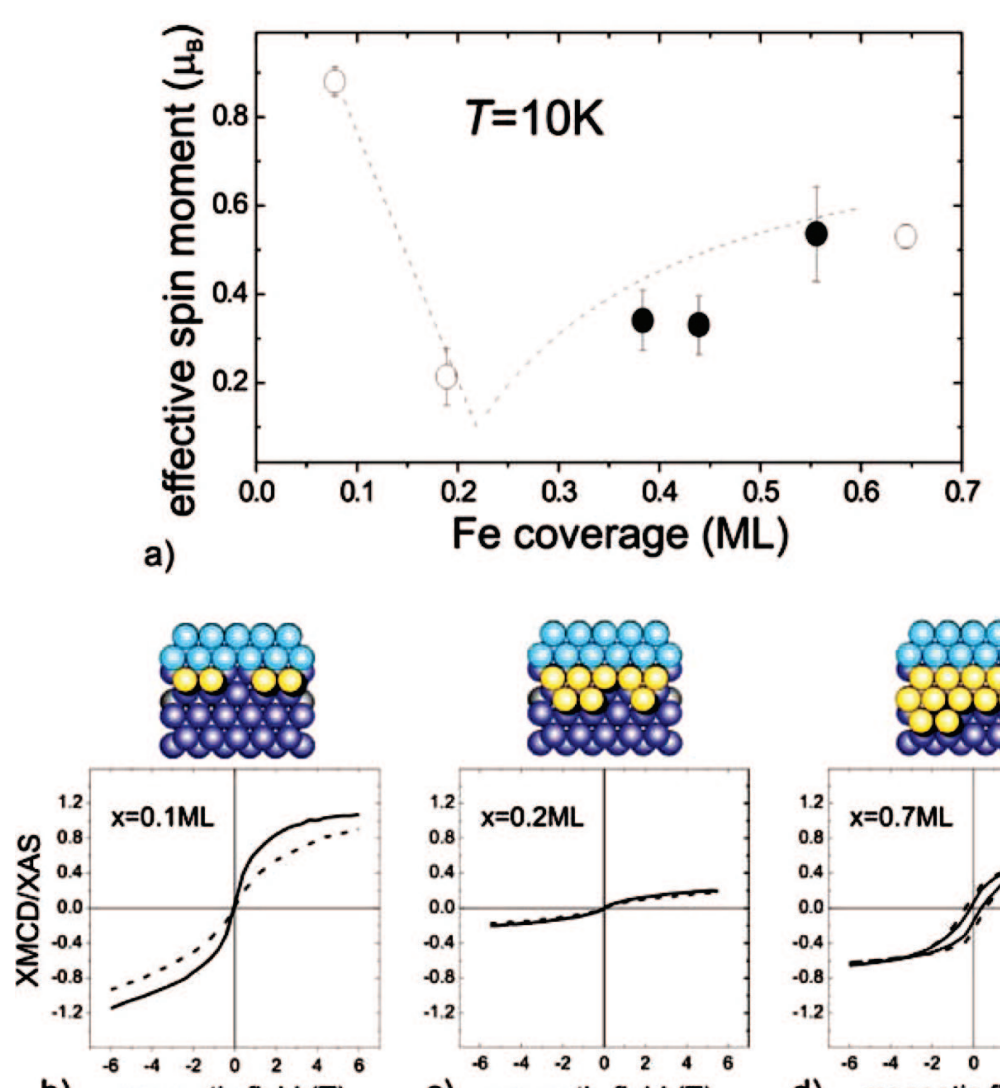

b) magnetic field ( $\mathrm{T}$ )

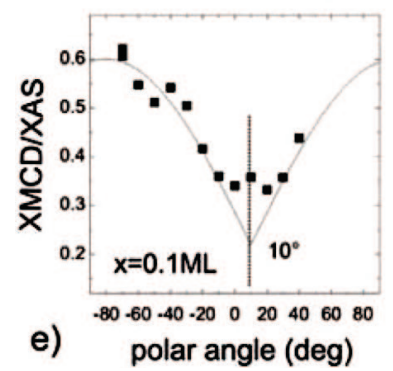

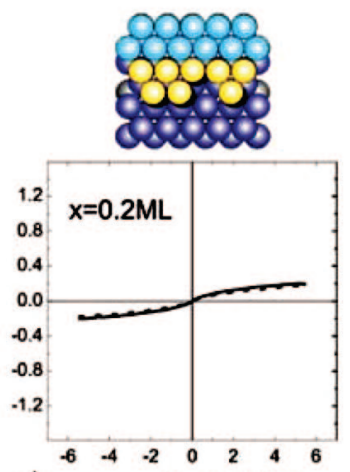

c) magnetic field (T)

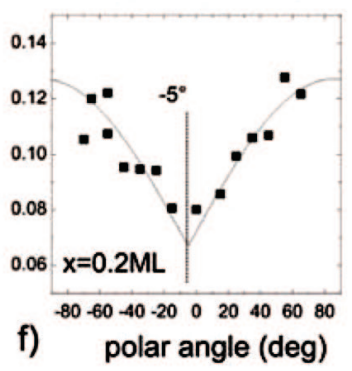

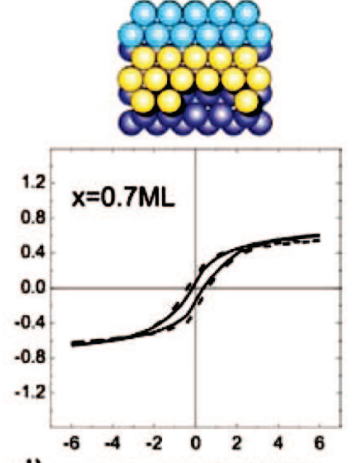

d) magnetic field (T)

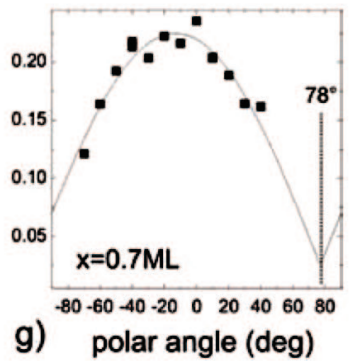

Figure 12. a) Average effective spin moments per atom and $3 d$ band hole measured in the magnetic easy axis directions at the respective coverages. The average moments are extracted from XMCD spectra measured at saturating fields of up to $6 \mathrm{~T}$ (the measurements were performed at ESRF (open symbols) and BESSY II (full symbols)). Central row: Polar (dashed lines) and in-plane (full lines) magnetization curves taken at $7 \mathrm{~K}$ for coverages: b) $x=0.1 \mathrm{ML}$, c) $0.2 \mathrm{ML}$ and d) $0.7 \mathrm{ML}$. Bottom row: Magnetization measurements performed $\mathrm{e}), \mathrm{g}$ ) in a field of $1 \mathrm{~T}$ and $\mathrm{f}$ ) in remanence for different directions with respect to the Pt(997) surface normal. The magnetization curves are extracted from XMCD data. Copyright (C) 2009 American Physical Society. Adapted with permission. ${ }^{47}$

miscut induces a periodic step structure, which forces the herringbone pattern to be uniformly oriented and homogeneous over macroscopic dimensions. We show in particular that the blocking temperature of Co islands arranged in a superlattice on $\mathrm{Au}(11,12,12)$ can significantly be enhanced by interfacing them with small amounts of Fe. The magnetic ground state of one island is FM order in a single domain. We present magnetic torque measurements revealing that also the transition state during thermal magnetization reversal is monodomain, i.e., the reversal takes place by coherent rotation of all magnetic moments. As a consequence, the blocking temperatures are directly proportional to the magnetic anisotropy energies.

In order to put these results into perspective we first briefly recall what has been reported on the growth and magnetic properties of pure Co islands on $\mathrm{Au}(788)$. Very regular superlattices of $\mathrm{Co}$ islands can be grown by self-assembly on $\mathrm{Au}(788) .{ }^{65}$ For deposition temperatures from 70 to $130 \mathrm{~K}^{66}$ nucleation takes place at the intersection of the surface partial dislocations of the reconstructed (111)-terraces ${ }^{67}$ and the monatomic steps. The steps repel each other by substrate mediated elastic interactions. ${ }^{68}$ This is important as it prevents step bunching, i.e., the steps stay monatomic, and for a perfectly clean crystal they are regularly spaced over mesoscopic distances. In addition, the partial dislocations are aligned between adjacent terraces leading to a rectangular lattice of nucleation sites phase-coherently covering the entire crystal. The resulting island superlattice has a period of $72 \AA$ along, and $38 \AA$ perpendicular to the steps, equivalent to a density of 24 Tera islands in ${ }^{-2}$. This is two orders of magnitude larger than the last generation of commercial hard-disks having a storage density of 200 Giga bits in ${ }^{-2}$ and far above the one of current magnetic recording media tested in the laboratories having feature densities of 1 Tera bits in ${ }^{-2}$. This makes stable Au vicinal surfaces ideal templates for the bottom-up fabrication of magnetic superlattices exploring the ultimate physical density limits of magnetic recording.

The magnetic properties of $\mathrm{Co} / \mathrm{Au}(788)$ have been characterized by means of XMCD, MOKE, and STM. ${ }^{69}$ The latter two techniques have been combined in the same UHV chamber in order to establish a one-to-one correlation between magnetism and morphology. ${ }^{43}$ The dependence of the orbital moments on field angle shows that the islands have uniaxial out-of-plane magnetic anisotropy. This magnetization direction minimizes dipolar interactions between the islands, that is one of the reasons why magnetic recording industry is moving to out-of-plane media. We found indeed that such interactions are negligible for the present system. A sensitive indicator is the decay of the zero-field susceptibility $x$ above the blocking temperature $T_{\mathrm{b}}$. For ensembles of noninteracting particles it is a little steeper than $1 / T, 43$ while a much more shallow decay has been predicted for interacting ones. ${ }^{70} \mathrm{Co} / \mathrm{Au}(788)$ followed exactly the non-interacting $\chi(T)$-curve until several times $T_{\mathrm{b}}$. Additional evidence for the absence of magnetic interactions has been derived from preparing a bi-modal size distribution for which the small and large islands show separate transitions from blocking to superparamagnetism, i.e., they do not influence each other. The maximum stray field acting on one island caused by its neighbors is estimated to $\mu_{\mathrm{B}} \mathrm{H}=40 \mathrm{mT}$, which can switch an island only when it is at $98 \%$ of its blocking temperature. 
(a)

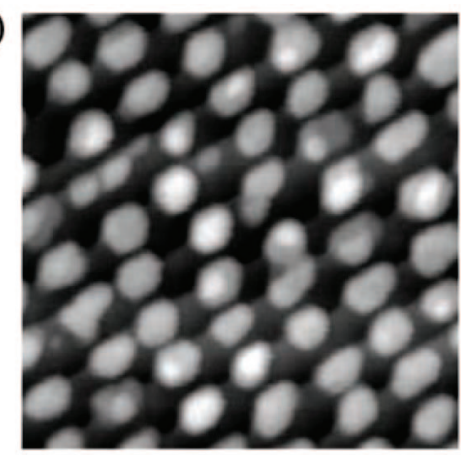

(c)

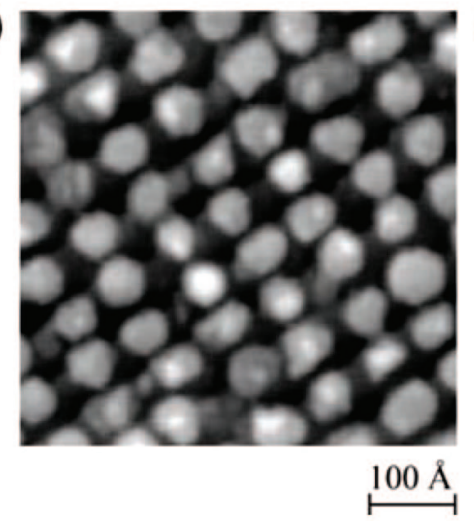

(b)

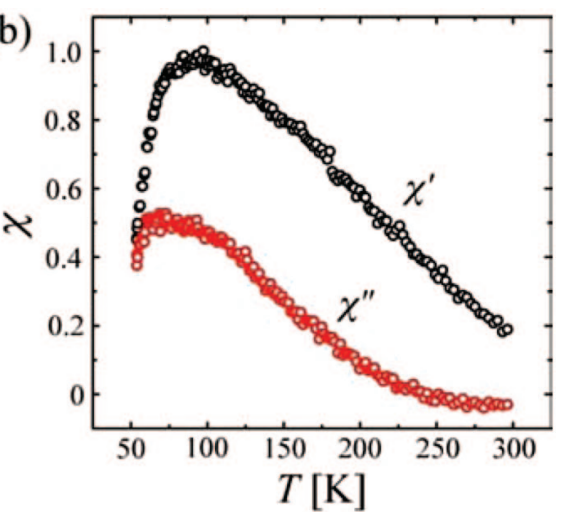

(d)

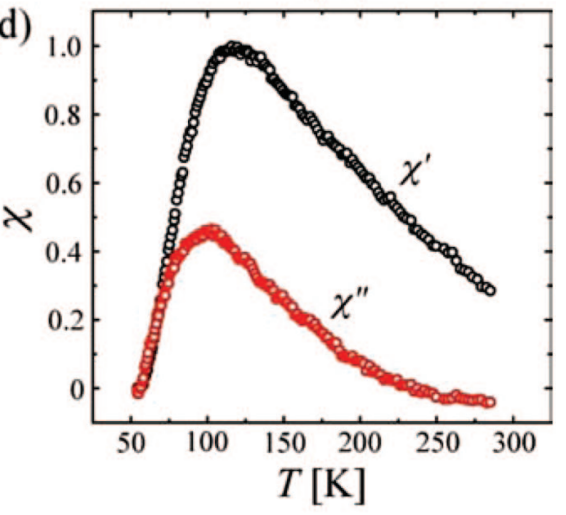

Figure 13. Increase in blocking temperature for a superlattice of magnetic islands on $\mathrm{Au}(11,12,12)$. a) STM image of pure Co clusters $\left(0.9 \mathrm{ML}, T_{\text {dep }}=130 \mathrm{~K}, T_{\text {ann }}=400 \mathrm{~K}\right)$. b) Real $\left(\chi^{\prime}\right.$, black) and imaginary ( $\chi^{\prime \prime}$ red) part of the zero-field susceptibility measured with MOKE for the sample shown in a). c) STM image of Co-core Fe-shell clusters ( $0.6 \mathrm{ML} \mathrm{Co}, 0.3 \mathrm{ML} \mathrm{Fe}, T_{\mathrm{Fe}}$ dep $=200 \mathrm{~K}, T_{\mathrm{Fe}-\mathrm{ann}}=300 \mathrm{~K}$ ). d) Temperature-dependent susceptibility showing the increase of the blocking temperature achieved by Fe decoration. For (b) and (d): field amplitude $100 \mathrm{G}$, modulation frequency $31 \mathrm{~Hz}$. For both STM images: $V_{\mathrm{t}}=1.7 \mathrm{~V}, I_{\mathrm{t}}=0.2 \mathrm{nA}, T=180 \mathrm{~K}$.

This shows that there is still some margin to increase the size, and possibly also the island density, without being limited by mutual interactions.

In addition, the distributions of the magnetic properties, such as switching field, anisotropy, and magnetic moment per island, are very narrow. As an example, the anisotropy $K$ has a half-width at half maximum (HWHM) of $17 \%$ which is by a factor of two more narrow than the most narrow anisotropy distributions reported for colloidal nanoparticles. ${ }^{71}$ Also the temperature where the FM order within one island breaks down, which we refer to as the Curie temperature $T_{C^{\prime}}$ is far above the blocking temperature. The only weak point of this system are the low blocking temperatures, only $45 \mathrm{~K}$ have been achieved for a superlattice with a mean island size of 300 atoms.

As we will show, this issue has been improved for superlattices grown on $\mathrm{Au}(11,12,12)$. Due to the smaller miscut from the crystallographic (111) direction, the terraces of this surface are wider and the superlattice has a period of $72 \AA \times 58 \AA$ and a density of 15 Tera islands in ${ }^{-2} .{ }^{72-75}$ This permits a slight increase in island size before the onset of coalescence and offers more flexibility for island decoration with other elements, again without starting morphological or magnetic coalescence.

The highest magnetic anisotropy energies per atom are achieved in bimetallic alloys with a crystal structure, such as the fct $L 1_{0}$ phase of FePt, implying a layerwise alternation of both elements. This strongly suggests that interfaces contribute significantly to the anisotropy. Often these alloys combine a magnetic element with a nonmagnetic one that is highly polarizable and has high SOC. Nanoparticles of such alloys combine the high anisotropy of the alloy with the abundance of low-coordinated atoms, which have been demonstrated to display large orbital magnetism with the associated large magnetic anisotropy. ${ }^{5}, 6,43$ Recently, we have demonstrated that also combining two magnetic $3 d$ elements, namely $\mathrm{Fe}$ and $\mathrm{Co}$, in a monolayer and in monolayer high clusters on $\mathrm{Pt}(111)$ results in strongly increased magneto-crystalline anisotropy. ${ }^{76}$ For this reason we created superlattices of islands with a lateral interface between these two elements on $\mathrm{Au}(11,12,12)$. The morphology and magnetic properties of Co-core Fe-shell clusters are compared in Figure 13 with the ones of pure Co islands of identical size.

Figure 13a shows a typical pattern of Co double layer islands obtained by deposition of $0.9 \mathrm{ML}$ onto $\mathrm{Au}(11,12,12)$ in $\leq 0.3 \mathrm{ML}$ increments, each deposition step at $130 \mathrm{~K}$ being followed by an annealing to $400 \mathrm{~K}$. The sequential deposition significantly improves the order with respect to the one obtained by room temperature deposition in a single step, ${ }^{72-74}$ and it pushes the onset of coalescence to higher coverages, as has been shown before on $\mathrm{Au}(788) .{ }^{68}$ For the present system the highest coverage before the onset of coalescence is 1.1 $\mathrm{ML}$, where the mean size is 600 atoms and the size distribution has $27 \%$ HWHM. ${ }^{75}$ The example shown in Figure 13a is thus well before the onset of coalescence. The clusters have a blocking temperature of $T_{\mathrm{b}}=75 \mathrm{~K}$, as can be inferred from the maximum of the imaginary part of the zero-field susceptibility measured with MOKE and shown in Figure 13b.

An STM image of a superlattice of bimetallic islands consisting of a Co core surrounded by an Fe shell is shown in Figure 13c. The island cores have been grown sequentially at the same deposition and annealing temperatures as above, while decoration with $\mathrm{Fe}$ has been done at $200 \mathrm{~K}$ followed by annealing to $300 \mathrm{~K}$. The morphology of this sample is hard to distinguish from the one of the pure Co islands, however, their blocking temperature is $T_{\mathrm{b}}=105 \mathrm{~K}$. This $40 \%$ increase is attributed to a pure electronic structure effect related to the Co-Fe hybridization at the core-shell interface. Without this hybridization, adding just $\mathrm{Fe}$ is expected to give rise to a decrease of the Co out-of-plane anisotropy since Fe islands on $\mathrm{Au}(111)$ have in-plane anisotropy. ${ }^{76}$ For CoFe films on $\mathrm{Pt}(111)$ we have found three times larger anisotropy than for pure $\mathrm{Co}$ and Fe. ${ }^{77}$ Electronic structure calculations revealed for this system that this is a consequence of the fine tuning of the occupation number of spin-down $\mathrm{d}_{x-y}^{2}{ }^{2}$ and $\mathrm{d}_{x y}$ in-plane orbitals, both for $\mathrm{Fe}$ and $\mathrm{Co}$, while the occupation of the $\mathrm{d}_{3 z_{7 j}}{ }^{2}$ ${ }_{r}^{2}, \mathrm{~d}_{x z}$ and $\mathrm{d}_{y z}$ out-of-plane orbitals remains nearly stable. ${ }^{7 \overline{7}}$ 
Likely, very similar electronic hybridization takes place at the Fe-Co interface in the bimetallic islands.

$\mathrm{Au}$ decoration of Co double layer islands on $\mathrm{Au}(111)$ has been reported to give rise to an increase in magnetic anisotropy by 5 to $11 \%$, depending on the initial Co coverage $(0.35$ vs. $1.15 \mathrm{ML}$ ); for a given Co coverage, this increase has its maximum at 0.4 to $0.5 \mathrm{ML} \mathrm{Au}$ from where on the magnetic anisotropy decreases until below the pure Co value. ${ }^{78}$ Right at the anisotropy maximum a transition from lateral to vertical decoration by Au takes place. The increase is small compared to the above results and has been attributed to strain inside the Co islands being modified by the Au shell. Strain is unlikely to be the main cause of anisotropy increase for the present case since Co and Fe have similar misfit with Au. But also for the $\mathrm{Au} / \mathrm{Co}$ system an alternative explanation emerges from XMCD results on spherical Co core Au shell nanoparticles showing large orbital moments induced in the $\mathrm{Au}$ atoms located at the interface with Co. ${ }^{79}$ This induced orbital moment is shown to have strong anisotropy entailing by SOC the large magnetic anisotropy energy of the overall magnetic moments.

A more direct access to the magnetic anisotropy than the temperature dependent zero-field susceptibility shown above can be gained by acquiring magnetization loops below the blocking temperature and at different field angles. We built a combined variable-temperature STM-MOKE set-up

(a)
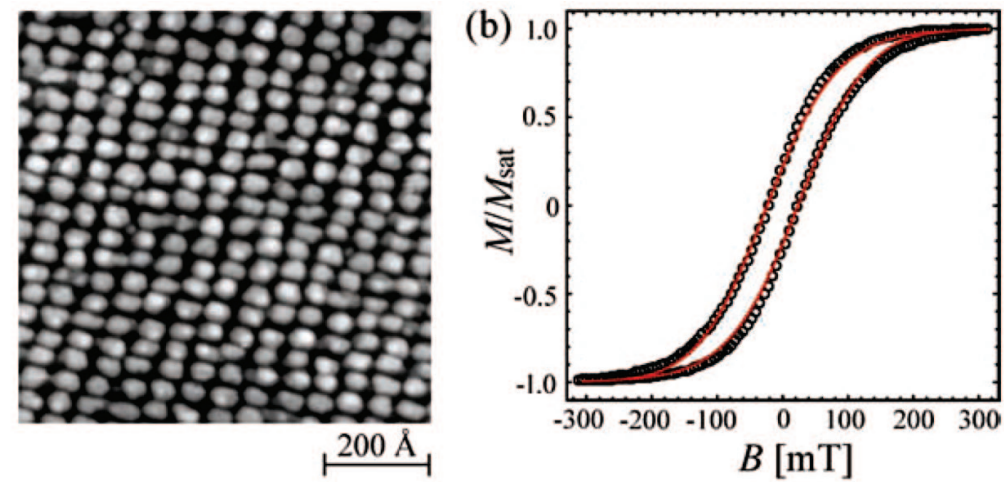

(c)
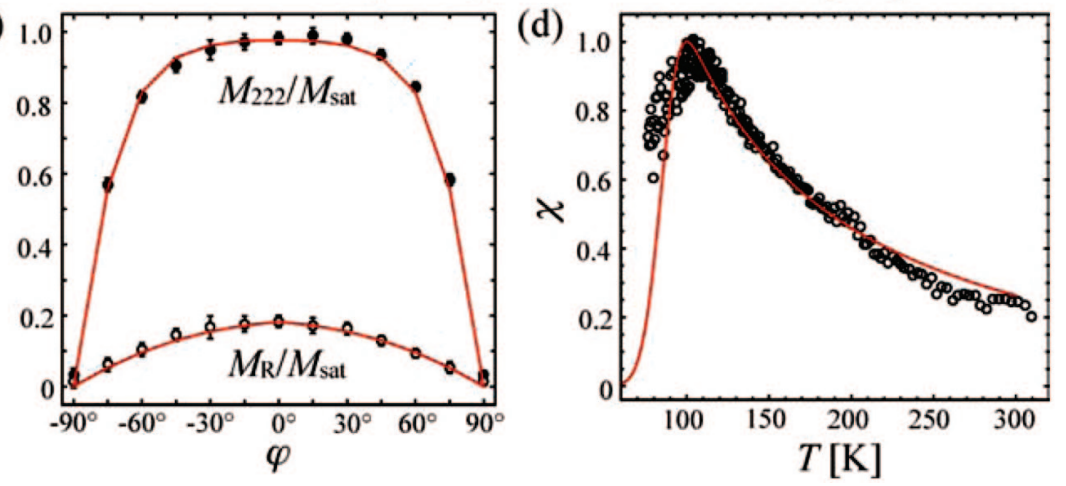

Figure 14. Magnetic torque measurement of anisotropy against blocking temperature derived from $\chi(T)$ for $1.1 \mathrm{MLCo} / \mathrm{Au}(11,12,12)$. a) STM image of the island array $\left(V_{\mathrm{t}}=1.8\right.$ $\mathrm{V}_{1} I_{\mathrm{t}}=0.2 \mathrm{nA}, T=200 \mathrm{~K}$ ). b) Magnetization loop measured at $T=75 \mathrm{~K}$ in polar geometry (field sweep rate $1.0 \mathrm{~T} \mathrm{~min}^{-1}$, field maximum $\mu_{0} \mathrm{H}=222 \mathrm{mT}$, average over two loops, error bar is smaller than symbol size and therefore not shown). c) Magnetization at $B=$ $222 \mathrm{mT}$ (full circles) and remanent magnetization (empty circles) measured at $T=75 \mathrm{~K}$. d) $\chi(T)=\left(\chi_{\text {Real }}^{2}+\chi_{I m}^{2}\right)^{1 / 2}$ measured by applying a triangular field sweep with a sweep rate of $13 \mathrm{~Hz}$ and $5 \mathrm{mT}$ amplitude. Solid red curves in (b-d) show best fits with the anisotropy per perimeter atom as only fit parameter. Copyright (c) 2009 American Institute of Physics. Adapted with permission. ${ }^{75}$ with a quadrupole magnet enabling transverse and polar Kerr measurements at any field orientation in the plane perbeams. ${ }^{75}$ Figure 14a shows the STM image for a sample with 1.1 ML $\mathrm{Co} / \mathrm{Au}(11,12,12)$ prepared the way described wove. Again, the order is very good and coalescence is not $14 \mathrm{~b}$. It reveals remanence and is rounded since some of the The angular dependence of the magnetization at recorded at $75 \mathrm{~K}$. The out-of-plane anisotropy is evident.

In order to derive anisotropy energies the mean value of the out-of-plane projected magnetization of the island surlattice in the presence of a time varying field of any angith respect to the surface normal has been modeled present knowledge for $\mathrm{Co} / \mathrm{Pt}(111), 6,43$ we assume the magnetic anisotropy energy to be proportional to the perimeter length $K=p K_{p}$, where $p$ is the number of perimeter atoms in the first and second layer. We further assume reasonable values for the attempt frequency, $v_{0}=10^{10} \mathrm{~Hz}$, and for the magnetic moment, $m=2.0 \mu_{\mathrm{B}}$ per atom and take the island size and perimeter length distribution experimentally determined from STM images, such as the one shown in Figure 14a into account. The single remaining fit parameter is then $K_{\mathrm{p}}$ for which we obtain $K_{p}=0.87 \pm 0.01 \mathrm{meV}$ per perimeter atom as the best fit of the experimental curves which are shown as full red lines in Figures $14 \mathrm{~b}, \mathrm{c}$. This anisotropy value is obtained when the islands are close to their ground state which is FM and monodomain. The small torque applied by the field does not favor the creation of domain walls and therefore this measurement is as close as one can get to the real anisotropy of a nanostructure.

$x(T)$ measurements are only then a direct measurement of $K$ if thermal magnetization reversal takes place by coherent rotation, i.e., if the transition state is monodomain. Experimentally, the transition state is difficult to access since it lasts very short and requires the spatial resolution to see a domain wall in one of the islands measuring only $3 \mathrm{~nm}$ in diameter. Therefore we access the nature of the transition state indirectly by comparing $\chi(T)$ with the $K_{\mathrm{p}}$-result obtained above for the very same sample in its magnetic ground state. Figure $14 \mathrm{~d}$ shows $\chi(T)$ measured while cooling. The solid red line is the best fit calculated using the macrospin model expressed in a previous report (Equation 1 and 2). ${ }^{43}$ with identical values for $v_{0}, m$, and using again the experimental island size and perimeter length distributions. The fit is very good and yields $K_{p}=0.95 \pm 0,01 \mathrm{meV}$ per perimeter atom. Both methods give very similar anisotropy energies. This shows that the macrospin model describes the present double 
layer islands with 600 atoms average size very well and the transition state during thermal magnetization reversal is monodomain for the present system. The $K$ values also compare very well with the ones found in earlier publications. ${ }^{69,80,81}$

In summary, Co island superlattices with uniaxial outof-plane magnetization, negligible mutual magnetic interactions, and narrow distributions of magnetic anisotropies, moments and switching fields can be synthesized by kinetically controlled atomic beam growth on Au vicinal surfaces. The magnetization reversal of these islands takes place by coherent rotation of all moments. The highest blocking temperatures so far achieved are $85 \mathrm{~K}$ for pure Co islands on $\mathrm{Au}(11,12,12)$ containing on average 600 atoms, and $105 \mathrm{~K}$ for Co core Fe shell islands with a mean size of only 490 atoms. By going closer to the coalescence threshold this value can possibly be increased to $125 \mathrm{~K}$. Further increase at similar densities will require growth into the third dimension which will also bring the benefit of the shape anisotropy going into the right direction.

\section{Conclusions}

We have shown that self-assembly methods can be used to construct dense arrays of 0D, 1D and 2D magnetic units with tunable magnetic properties and dimensions that approach the ultimate atomic limit. It is quite evident that a mere extrapolation from the classical and microscale behavior is generally entirely inadequate for describing magnetic structures approaching the atomic size. Ab initio electronic structure calculations have been used to highlight Fermi surface and spinorbit coupling effects that become prominent at the nanoscale. The exploration of magnetism in subnanometer scale materials has revealed a wealth of notable and unusual properties, such as complex magnetic phases, symmetry-allowed Dzyaloshinskii-Moriya exchange, enhanced moments, magnetic anisotropy, and spin reorientation transitions. Here we have emphasized: 1) the interaction of magnetic atoms with different nonmagnetic substrates, 2) their self-assembly into ordered two-dimensional superlattices with tunable magnetic properties, 3) the special role of orbital moments and SOC in determining the magnetic structure and stability of low coordination systems, such as 1D wires and 2D particles, and 4) the fabrication of very dense magnetic particle arrays using self-organized surface templates. Given the importance of thermal fluctuations in small magnetic particles, we have paid specific attention to methods that allow control of the magnetic anisotropy energy, hence of the magnetization orientation and thermal stability, in self-assembled systems. Spin reorientation transitions and large zero-field splitting can be induced either by charging or ligand-field manipulation in metal-organic complexes, even when these are coordinated to a metal surface. In all-metal particles, 2D arrays of bilayer Co structures can be realized with $6 \times 7 \mathrm{~nm}^{2}$ periodicity, uniaxial out-of-plane magnetization, and negligible mutual interactions. By assembling particles with a Co core and an Fe shell structure, we obtained enhanced magnetization properties as well as an increase of the blocking temperature with respect to pure Co particles of equal size. Although self-assembled systems do not yet match the uniformity and temperature stability of lithographic magnetic memories, they present superior areal densities and uncommon magnetic properties that serve the development of new materials and concepts in the fields of nanoelectronics and spintronics. Our studies illustrate that extreme flexibility in the engineering of functional properties can be achieved through atomic-scale design of the magnetic elements. Accurate control of the geometry and internal structure of nanosized magnetic particles, combined with deep understanding of the underlying physical interactions, is the key issue for bringing them into the realm of practical operation.

Acknowledgments - We acknowledge support from the European Science Foundation (EUROCORES Programme SONS under contract N. ERAS-CT-2003-980409) and the respective national science foundations. S.V. has been supported by the Swiss National Science Foundation (129934). S.S., C.K., T.B., A.M., and P.G. have been supported by the Spanish Ministerio de Ciencia e Innovación (MAT200762341), the Catalan Agéncia de Gestió d'Ajuts Universitaris i de Recerca (2009 SGR 695), and the European Research Council (StG 203239 NOMAD). A.E. is supported by NSF grants DMR-0747704 and DRM-0213808. Provision of beamtime and experimental support from the European Synchrotron Radiation Facility is gratefully acknowledged. This article is part of a Special Issue on Multiscale Self-Organization of Functional Nanostructures.

\section{References}

[ 1 ] A. Wolf, D. D. Awschalom, R. A. Buhrman, J. M. Daughton, S. von Molnár, M. L. Roukes, A. Y. Chtchelkanova, D. M. Treger, Science 2001, 294, 1488 .

[ 2 ] A. Allwood, G. Xiong, C. C. Faulkner, D. Atkinson, D. Petit, R. P. Cowburn, Science 2005, 309, 1688 ; b) A. Ney, C. Pampuch, R. Koch, K. H. Ploog, Nature 2003, 425, 485.

[ 3 ] J. V. Barth, G. Costantini, K. Kern, Nature 2005, 437, 671.

[ 4 ] D. A. Thomson, J. S. Best, IBM J. Res. Dev. 2000, 44, 311 ; S. D. Bader, Rev. Mod. Phys. 2006, 78, 1.

[ 5 ] P. Gambardella, A. Dallmayer, K. Maiti, M. C. Malagoli, W. Eberhardt, K. Kern, C. Carbone, Nature 2002, 416, 301.

[ 6 ] P. Gambardella, S. Rusponi, M. Veronese, S.S. Dhesi, C. Grazioli, A. Dallmeyer, I. Cabria, R. Zeller, P. H. Dederichs, K. Kern, C. Carbone, H. Brune, Science 2003, 300, 1130 .

[ 7 ] a) M. A. Ruderman, C. Kittel, Phys. Rev. 1954, 96, 99 ; b) T. Kasuya, Prog. Theor. Phys. 1956, 16, 45; c) K. Yosida, Phys. Rev. 1957, 106, 893.

[ 8 ] L. M. Roth, H. J. Zeiger, T. A. Kaplan, Phys. Rev. 1966, 149, 519.

[ 9 ] P. Grünberg, R. Schreiber, Y. Pang, M. B. Brodsky, H. Sower, Phys. Rev. Lett. 1986, 57, 2442.

[ 10 ] S. S. P. Parkin, N. More, K. P. Roche, Phys. Rev. Lett. 1990, 64, 2304.

[ 11 ] P. Bruno, C. Chappert, Phys. Rev. Lett. 1991, 67, 1602.

[ 12 ] a) A. Weismann, M. Wenderoth, S. Lounis, P. Zahn, N. Quaas, R. G. Ulbrich, P. H. Dederichs, S. Blügel, Science 2009, 323, 1190 ; b) A. J. Heinrich, Science 2009, 323, 1178.

[ 13 ] F. Meier, L. Zhou, J. Wiebe, R. Wiesendanger, Science 2008, 320, 82.

[ 14 ] L. Zhou, J. Wiebe, S. Lounis, E. Vedmedenko, F. Meier, S. Blügel, P. H. Dederichs, R. Wiesendanger, Nat. Phys. 2010, 6, 187.

[ 15 ] N. Papanikolaou, R. Zeller, P. H. Dederichs, J. Phys. Condens. Matter 2002, 14, 2799

[ 16 ] J. Tersoff, D. R. Hamann, Phys. Rev. Lett. 1983, 50, 1998.

[ 17 ] M. Bode, M. Heide, K. v. Bergmann, P. Ferriani, S. Heinze, G. Bihlmayer, A. Kubetzka, O. Pietzsch, S. Blügel, R. Wiesendanger, Nature 2007, 447, 190.

[ 18 ] P. Ferriani, K. v. Bergmann, E. Y. Vedmedenko, S. Heinze, M. Bode, M. Heide, G. Bihlmayer, S. Blügel, R. Wiesendanger, Phys. Rev. Lett. 
2008, 101, 027201

[ 19] M. Heide, G. Bihlmayer, S. Blügel, Phys. Rev. B 2008, 78, 140403(R).

[ 20 ] Ph. Kurz, F. Förster, L. Nordström, G. Bihlmayer, S. Blügel, Phys. Rev. B 2004, 69, 024415.

[ 21 ] M. Heide, G. Bihlmayer, S. Blügel, Physica B 2009, 404, 2678.

[ 22 ] O. Pietzsch, A. Kubetzka, M. Bode, R. Wiesendanger, Science 2001, 92, 2053.

[ 23 ] N. Atodiresei, P. H. Dederichs, Y. Mokrousov, L. Bergqvist, G. Bihlmayer, S. Blügel, Phys. Rev. Lett. 2008, 100, 117207.

[ 24 ] H. Brune, P. Gambardella, Surf. Sci. 2009, 03, 1830.

[ 25 ] P. Gambardella, S. S. Dhesi, S. Gardonio, C. Grazioli, P. Ohresser, C. Carbone, Phys. Rev. Lett. 2002, 88, 047202.

[ 26 ] P. Gambardella, H. Brune, S. S. Dhesi, P. Bencok, K. Menon, S. Gardonio, M. Veronese, C. Grazioli, C. Carbone, Phys. Rev. B 2005, 72, 045337

[ 27 ] P. Gambardella, S. Rusponi, T. Cren, N. Weiss, H. Brune, C. R. Physique 2005, 6, 75 .

[ 28 ] P. Blonski, A. Lehnert, S. Dennler, S. Rusponi, M. Etzkorn, G. Moulas, P. Bencok, P. Gambardella, H. Brune, J. Hafner, Phys. Rev. B 2010, 81, 104426

[ 29 ] A. J. Heinrich, J. A. Gupta, C. P. Lutz, D. M. Eigler, Science 2004, 306,466 .

[ 30 ] C. F. Hirjibehedin, C.-Y. Lin, A. F. Otte, M. Ternes, C. P. Lutz, B. A. Jones, A. J. Heinrich, Science 2007, 317, 1199.

[ 31 ] O. Yu. Kolesnichenko, R de Kort, M. I. Katsnelson, A. I. Lichtenstein, van H. Kempen, Nature 2002, 415, 507.

[ 32 ] G. Racah, Phys. Rev. 1942, 62, 438.

[ 33 ] E. U. Condon, G. H. Shortly, The Theory of Atomic Spectra, Cambridge Univ. Press, London, 1935.

[ 34 ] A. C. Hewson, The Kondo Problem to Heavy Fermions, Cambridge Univ. Press, London, 1993

[ 35 ] V. Madhavan, W. Chen, T. Jamneala, M. F. Crommie, N. S. Wingreen, Science 1998, 280, 569

[ 36 ] J. Li, W. Schneider, R. Berndt, B. Delley, Phys. Rev. Lett. 1998, 80, 2893.

[ 37 ] C. Carbone, M. Veronese, P. Moras, S. Gardonio, C. Grazioli, P. H. Zhou, O. Rader, A. Varykhalov, C. Krull, T. Balashov, A. Mugarza, P. Gambardella, S. Lebeque, O. Eriksson, M. I. Katsnelson, A. I. Lichtenstein, Phys. Rev. Lett. 2010, 104, 117601.

[ 38 ] M. Ternes, A. J. Heinrich, W.-D. Schneider, J. Phys. Condens. Matter 2009, 21, 053001 .

[ 39 ] V. S. Stepanyuk, W. Hergert, K. Wildberger, R. Zeller, P. H. Dederichs, Phys. Rev. B 1996, 53, 2121

[ 40 ] B. Lazarovits, L. Szunyogh, P. Weinberger, Phys. Rev. B 2003, 67, 024415.

[ 41] O. Sipr, S. Bornemann, J. Minár, S. Polesya, V. Popescu, A. Simunek, H. Ebert, J. Phys. Condens. Matter 2007, 19, 096203.

[ 42 ] G. van der Laan, B. T. Thole, Phys. Rev. B 1991, 43, 13401.

[ 43 ] S. Rusponi, T. Cren, N. Weiss, M. Epple, P. Buluschek, L. Claude, H. Brune, Nature Mater. 2003, 2, 546

[ 44 ] P. Gambardella, A. Dallmeyer, K. Maiti, M. C. Malagoli, S. Rusponi, P. Ohresser, W. Eberhardt, C. Carbone, K. Kern, Phys. Rev. Lett. 2004, 93, 077203

[ 45 ] P. Gambardella, J. Phys.: Condens. Matter 2003, 15, S2533.

[ 46] A. Vindigni, A. Rettori, M. G. Pini, C. Carbone, P. Gambardella, Appl. Phys. A 2006, 82, 385.

[ 47 ] J. Honolka, T. Y. Lee, K. Kuhnke, D. Repetto, V. Sessi, P. Wahl, A. Buchsbaum, P. Varga, S. Gardonio, C. Carbone, S. R. Krishnakumar, P. Gambardella, M. Komelj, R. Singer, M. Faehnle, K. Fauth, G. Schuetz, A. Enders, K. Kern, Phys. Rev. B 2009, 79, 104430.

[ 48 ] P. Gambardella, S. Stepanow, A. Dmitriev, J. Honolka, F. de Groot, M. Lingenfelder, S. Sen Gupta, D. D. Sarma, P. Bencok, S. Stanescu, S. Clair, S. Pons, N. Lin, A. P. Seitsonen, H. Brune, J. V. Barth, K. Kern, Nat. Mater. 2009, 8, 189.

[ 49 ] S. Stepanow, A. Mugarza, G. Ceballos, P. Moras, J. Cezar, C. Carbone, P. Gambardella, Phys. Rev. B 2010, 82, 014405.

[ 50 ] A. Mugarza, N. Lorente, P. Ordejon, C. Krull, S. Stepanow, M.-L.
Bocquet, J. Fraxedas, G. Ceballos, P. Gambardella, Phys. Rev. Lett. 2010, 105, 115702 .

[ 51 ] S. Stepanow, J. Honolka, P. Gambardella, L. Vitali, N. Abdurakhmanova, T.-C. Tseng, S. Rauschenbach, S. L. Tait, V. Sessi, S. Klyatskaya, M. Ruben, K. Kern, J. Am. Chem. Soc. 2010, 132, 11900

[ 52 ] S. Stepanow, N. Lin, J. V. Barth, J. Phys. Condens. Matter 2008, 20, 184002.

[ 53 ] S. Stepanow, M. Lingenfelder, A. Dmitriev, H. Spillmann, E. Delvigne, N. Lin, X. Deng, C. Cai, J. V. Barth, K. Kern, Nat. Mater. 2004, 3, 229.

[ 54 ] M. A. Lingenfelder, H. Spillmann, A. Dmitriev, S. Stepanow, N. Lin, J. V. Barth, K. Kern, Chem. Eur. J. 2004, 10, 1913.

[ 55 ] R. K. Hocking, E. C. Wasinger, Y.-L. Yan, F. M. F. deGroot, F. A. Walker, K. O. Hodgson, B. Hedman, E. I. Solomon, J. Am. Chem. Soc. 2007, 129, 113 .

[ 56 ] L. Bogani, W. Wernsdorfer, Nat. Mater. 2008, 7, 179.

[ 57 ] W. J. M. Naber, S. Faez, W. G. Van Der Wiel, J. Phys. D: Appl. Phys. 2007, 40, R205

[ 58 ] B. Hardrat, A. Al-Zubi, P. Ferriani, S. Blügel, G. Bihlmayer, S. Heinze, Phys. Rev. B 2009, 79, 094411.

[ 59 ] K. von Bergmann, S. Heinze, M. Bode, E. Y. Vedmedenko, G. Bihlmayer, S. Blügel, R. Wiesendanger, Phys. Rev. Lett. 2006, 96, 167203.

[ 60 ] T. Y. Lee, S. Sarbach, K. Kuhnke, K. Kern, Surf. Sci. 2006, 600, 3266

[ 61 ] M. Komelj, D. Steiauf, M. Fähnle, Phys. Rev. B 2006, 73, 134428.

[ 62 ] D. Repetto, T. Y. Lee, S. Rusponi, J. Honolka, K. Kuhnke, V. Sessi, U. Starke, H. Brune, P. Gambardella, C. Carbone, A. Enders, K. Kern, Phys. Rev. B 2006, 74, 054408

[ 63 ] G. Herzer, IEEE Trans. Magn. 1989, 25, 3327

[ 64 ] S. Uzdin, V. Uzdin, C. Demangeat, Europhys. Lett. 1999, 47, 556.

[ 65 ] V. Repain, G. Baudot, H. Ellmer, S. Rousset, Europhys. Lett. 2002, $58,730$.

[ 66 ] V. Repain, S. Rohart, Y. Girard, A. Tejeda, S. Rousset, J. Phys.: Condens. Matter 2006, 18, S17.

[ 67 ] J. V. Barth, H. Brune, G. Ertl, R. Behm, Phys. Rev. B 1990, $42,9307$.

[ 68 ] G. Prévot, B. Croset, Phys. Rev. Lett. 2004, 92, 256104.

[ 69 ] N. Weiss, T. Cren, M. Epple, S. Rusponi, G. Baudot, S. Rohart, A. Tejeda, V. Repain, S. Rousset, P. Ohresser, F. Scheurer, P. Bencok, H. Brune, Phys. Rev. Lett. 2005, 95, 157204.

[ 70 ] R. W. Chantrell, N. Walmsley, J. Gore, M. Maylin, Phys. Rev. B 2000, 63,024410

[ 71 ] S. I. Woods, J. R. Kirtley, S. Sun, R. H. Koch, Phys. Rev. Lett. 2001, 87,137205

[ 72 ] S. Rousset, V. Repain, G. Baudot, Y. Garreau, J. Lecoeur, J. Phys.: Condens. Matter 2003, 15, S3363.

[ 73 ] G. Baudot, S. Rohart, V. Repain, H. Ellmer, Y. Girard, S. Rousset, Appl. Surf. Sci. 2003, 212, 360

[ 74 ] N. Witkowski, Y. Borensztein, G. Baudot, V. Repain, Y. Girard, S. Rousset, Phys. Rev. B 2004, 70, 085408

[ 75] A. Lehnert, P. Buluschek, N. Weiss, J. Giesecke, T. Treier, S. Rusponi, H. Brune, Rev. Sci. Instrum. 2009, 80, 023902.

[ 76 ] P. Ohresser, N. Brookes, S. Padovani, F. Scheurer, H. Bulou, Phys. Rev. B 2001, 64, 104429

[ 77 ] G. Moulas, A. Lehnert, S. Rusponi, J. Zabloudil, C. Etz, S. Ouazi, M. Etzkorn, P. Bencok, P. Gambardella, P. Weinberger, H. Brune, Phys. Rev. B 2008, 78, 214424

[ 78 ] Y. Nahas, V. Repain, C. Chacon, Y. Girard, J. Lagoute, G. Rodary, J. Klein, S. Rousset, H. Bulou, C. Goyhenex, Phys. Rev. Lett. 2009, 103, 067202 .

[ 79 ] J. Bartolomé, L. M. García, F. Bartolomé, F. Luis, R. López-Ruiz, F. Petroff, C. Deranlot, F. Wilhelm, A. Rogalev, P. Bencok, N. B. Brookes, L. Ruiz, J. M. González-Calbet, Phys. Rev. B 2008, 77, 184420 .

[ 80 ] H. A. Dürr, S. S. Dhesi, E. Dudzik, D. Knabben, G. Van Der Laan, J. B. Goedkoop, F. U. Hillebrecht, Phys. Rev. B 1999, 59, R701

[ 81 ] S. Rohart, V. Repain, A. Tejeda, P. Ohresser, F. Scheurer, P. Bencok, J. Ferr, S. Rousset, Phys. Rev. B 2006, 73, 165412. 